\title{
Flexible inverse adaptive fuzzy inference model to identify the evolution of Operational Value at Risk for improving operational risk management
}

\author{
Alejandro Peña ${ }^{\mathrm{a}, *}$, Isis Bonet ${ }^{\mathrm{a}}$, Christian Lochmuller $^{\mathrm{a}}$, Francisco Chiclana ${ }^{\mathrm{b}}$, Mario Góngora $^{\mathrm{b}}$ \\ ${ }^{a}$ Research Group: Computational Modeling and Simulation (GISMOC and GPC), EIA University, Envigado, Colombia \\ ${ }^{b}$ Centre for Computational Intelligence (CCI), De Montfort University, Leicester, England
}

\begin{abstract}
Operational risk was one of the most important risks in the 2008 global financial crisis. This is due to limitations of the applied models in explaining and estimating this type of risk from highly qualitative information related to failures in the operations of financial organizations. A review of research literature on this area indicates an increase in the development of models for the estimation of the operational value at risk. However, there is a lack of models that use qualitative information for estimating this type of risk. Motivated by this finding, we propose a Flexible Inverse Adaptive Fuzzy Inference Model that integrates both a novel Montecarlo sampling method for the linguistic input variables of frequency and severity that allow the characterization of a risk event, the impact of risk management matrices to estimate the loss distribution and the associated operational value at risk. The methodology follows a loss distribution approach as defined by Basel II. A benefit of the proposed model is that it works with highly qualitative risk data and it also connects the risk measurement (operational value at risk) with risk management, based on risk management matrices. This way, we mitigate limitations related to a lack of available operational risk event data when assessing operational risk. We evaluate the experimental results obtained through the proposed model by using the Index of Agreement indicator. The results provide a flexible loss distribution under different risk profiles or risk management matrices that explain the evolution of operational risk in real time.
\end{abstract}

Keywords: operational risk, adaptive fuzzy inference model, Montecarlo sampling, loss distribution approach, operational value at risk, risk management matrix, Basel Committee on Banking Supervision, Basel II.

\section{Introduction}

Operational Risk was one of the most important risks in the 2008 global financial crisis as the applied models have limitations in explaining and estimating this type of risk from highly qualitative information related to failures in the operations of a business and, in particular, in operations of financial organizations. According to the Basel II agreement, established through the Basel Committee on Banking Supervision (BCBS), operational risk (OpRisk) is defined as "the risk of loss resulting from inadequate or failed internal processes, people and systems or from external events" [1]. This means that this type of risk occurs when business processes and units do not perform well and when different qualitative elements are involved, which are not static and evolve over time.

The operational value at risk $(\mathrm{OpVaR})$ is defined as the maximum loss which can be expected given a specific confidence level $(\alpha)$ in a certain period of time. This value can be estimated based on a loss distribution (LD) ) which can be built using the frequency and severity of a set of operational risk events generated within a certain period of time by OpRisk events in a particular business line associated with a particular risk type, such as fraud for example $[2,3]$. However, when it comes to measuring OpRisk, one problem is that compared with other types of risk data, like credit or market

\footnotetext{
${ }^{*}$ Corresponding author

Email addresses: fjapena@eia.edu.co (Alejandro Peña), isis.bonet@eia.edu.co (Isis Bonet), christian.lochmuller@eia.edu.co (Christian Lochmuller), chiclana@dmu.ac.uk (Francisco Chiclana), mgongora@dmu.ac.uk (Mario Góngora)
} 
risk data, operational risk data is not that frequent. Moreover, , according to [4], the OpRisk sources and exposures are more diverse, complex and context-dependent than those typical of market and credit risk. That is why supervisors require that the measurement of OpRisk also includes qualitative methods, such as scenario analysis, Risk and Control Self Assessments (RCSA) or Key Risk Indicators [5].

In the area of Advanced Measurement Approach (AMA) models, as defined by Basel II, four research development trends that focus on estimating the LD to estimate OpVaR have emerged:

1. A first line of research refers to the development of Bayesian risk models that aim to identify the causes, the influence and the relations between a set of factors that define the risk exposure of an organization or financial institution, where the relationship between events that are used to estimate the OpVaR are relevant for statistical evaluation $[6,7,8,9]$.

2. A second line of research focuses on vector models that adapt and learn from OpRisk data. These models allow to identify factors, parameters and variables that are relevant to model OpRisk. Within this research area, it is worth mentioning support vector machines (SVMs) integrating various classifiers [10], using multiple agent systems for learning [11], using experimental designs for selecting optimal weights [12], or applying Bayesian concepts to identify the causes and influence between risk factors $[13,14]$. These models allow to estimate the OpVaR using quantitative relationships between the data.

3. A third line of research is based on the principles of modeling and simulation of OpRisk. Here, we find models that allow to identify qualitatively the variables and parameters that can be used to model OpRisk and estimate the OpVaR through the use of ontologies [15] or that identify relevant information by using data mining techniques [16]; auto-regressive models for making predictions, based on the evolution of the data, to estimate the OpVaR for both short and medium time predictions $[17,18,19]$, as well as models that use multivariate distributions based on copulas to obtain the $\operatorname{LD}[20,2,21,22]$.

4. Due to the complexity of the factors that involve OpRisk and the big volume of qualitative data associated, a fourth line of research focuses on risk models that apply the principles of intelligent computational systems. Among these models, fuzzy systems stand out as they have demonstrated their effectiveness assessing risk in areas such like aviation or nutritional security [23]. Also, we can find models to estimate risk based on fuzzy neural networks that use different learning schemes $[24,25]$ or linguistic variables [26, 27, 28, 29].

Financial information is obtained from both public and private institutions as well as from experts with great knowledge and experience. In line with the aforementioned development trends, it is worth noting that different analytical models to estimate OpVaR work with crisp information. However, there also exist economic factors outside their control which are related to the impact and management of failures in business processes that include intuition and feelings expressed by experts that generate uncertainty [30]. This gives ground to the development of models that require the use of fuzzy and linguistic concepts to explain and estimate OpVaR and its evolution over time.

This paper focuses on the development of a Flexible Inverse Adaptive Fuzzy Inference Model (FIAFIm), framed within the fourth development line described above, to estimate the loss distribution (LD) and the associated OpVaR. FIAFIm integrates both a novel Montecarlo sampling method for fuzzy input variables and different risk management matrices (RMMs - risk profiles), which allows to assess how the operational risk in an organization or financial entity evolves. The flexible structure of FIAFIm takes three different forms in accordance with the degrees of freedom that define the structure of the model:

Model 1: Inverse Adaptive Uniform Fuzzy Inference Model (IAUFIm) to identify the risk management matrix (RMM) using balanced fuzzy sets for both frequency and severity of risk (to explain the risk profiles).

Model 2: Inverse Adaptive Non Uniform Fuzzy Inference Model (IANUFIm) to identify the RMM and the structure and shape of input and output fuzzy sets using adaptive unbalanced fuzzy sets (to build fuzzy input sets for frequency and severity). 
Model 3: Inverse Adaptive Sampling Fuzzy Inference Model (IASFIm) using fuzzy sets obtained by sampling Montecarlo method to analyze how risk evolves (to explain the evolution of risk over time).

For the setting, the model carried out a learning process with the goal to estimate the LD of reference (LD_MC), defined by the BCBS (learning phase), using a Montecarlo sampling method according to the AMA models. After the learning process and absence of learning mechanism (evolve phase), each of the models carried out the estimation of the LD for a sequence composed by three risk profiles or RMMs [E1 - strong impact (poor management); E2 - medium impact (moderate management); E3 - soft impact (strong management)] that conforms a natural sequence for managing risk in an organization or financial entity (risk evolution).

The benefit of the proposed model is that it works with highly qualitative risk data and also connects the risk measurement $(\mathrm{OpVaR})$ with risk management, based on the risk management matrices. Thus, this paper makes a contribution that goes beyond mere compliance with standards like Basel II by exploring new ways for operational risk management. The results obtained by the model in the learning phase for each of the structures (Model 1 to Model 3) show that the index of agreement (IOA) is close to one with regard to the LD_MC. To evaluate the structure and shape of the membership functions that define the input variables as fuzzy sets after the learning phase, a novel method of analysis is proposed that is based on the Q-Q Plot. The LD distributions estimated by the FIAFIm for each risk profile, which make up the sequence of the aforementioned risk in a second stage, show that the LD distribution evolves towards lower values of expected loss (EL), unexpected loss (UL) and OpVaR due to the effect of a better risk management. The structure (type of distribution) and shape (long tail) of the LDs was maintained, corroborating the stability of the model in estimating the OpVaR in absence of a learning process. Thus, the proposed model allows to obtain a flexible LD, integrating into a single model both a novel Montecarlo sampling method for fuzzy variables and the sequence of RMMs to explain a priori the evolution of OpVaR in real time. This makes the fuzzy model ideal to explain and estimate a priori the OpRisk over time, reducing the time required by AMA models to assess the impact of implementing the risk profile in a financial organization.

The rest of the paper is organized as follows: Section 2 presents the preliminary theory regarding the modeling of the linguistic variables of frequency and severity of a risk event, the methodologies to determine the regulatory capital for OpRisk, the estimation of the LD using the Montecarlo method, as well as the basic concepts that define the estimation of $O p V a R_{\alpha}$. Section 3 describes the methodology that supports the FIAFIm as well as its behavior with respect to the estimation of the LD. Section 4 reports the experimental results to assess the performance of the FIAFIm by using the loss distribution approach (LDA) applying different risk profiles. Finally, section 5 presents conclusions and future research work.

\section{Preliminary theory}

\subsection{Related work - a brief overview}

In addition to the development lines described in the introduction section, it can be observed that a range of risk modeling methods exist that vary in their relative reliance on historical data versus other inputs, such as expert opinion [31, 32]. Cruz, Peters and Shevchenko [33] give an extensive overview of operational risk modeling, assessment, and management techniques. Takács [34] applies fuzzy logic based risk management to disaster management, while Shang and Hossen [35] point out that fuzzy logic systems help simplify risks that do not have a proper quantitative probability model, as a fuzzy logic system can help model OpRisk, considering both the available data and experts' opinions. There exist alternative approaches to fuzzy logic models, such as Bayesian and artificial neural networks, hidden Markov and decision tree models, which Shang and Hossen [35] compare with regard to their strength and weaknesses when applied to risk management. Their work concludes that fuzzy logic models can be used as a complement to probability models and can be applied to assess risks for which there is insufficient data and incomplete knowledge, thus, linking their findings to the characteristics of OpRisk. Accordingly, soft computing techniques are applied in the field of operational risk management [4], although the Basel Accords do not mention them. Reveiz and León present in [4] a fuzzy inference 
system (FIS) as an alternative that "allows exploiting human (logic) reasoning and expert knowledge to articulate qualitative and quantitative inputs" when evaluating OpRisk. Reveiz and León use a 0 to 10 OpRisk Indicator, which relies on triangular membership functions to classify into the following qualitative levels of the indicator: negligible; negligible/low; very low; low; low/medium; medium/high; high; very high; catastrophic; high/catastrophic. Accordingly, "an expert (or group of experts) analyzes the Key Risk Indicators (KRIs), their different linkages and their relation to the linguistic variables in the output space, resulting in a list or set of educated inference rules that will solve simultaneously any combination of inputs and calculate the expected" OpRisk indicator [4]. However, these authors do not use OpVaR as an indicator or measure for modeling OpRisk. Mainelli [36] also instrument and present a Support Vector Machine example, contrasting a subset of actual versus predicted trade price bands. Sanford and Moosa [37] apply Bayesian networks to OpRisk and underline as a strength that Bayesian networks are good at modeling subjective and soft elements, such as subjective probabilities, sourced from domain experts. These authors apply the proposed Bayesian network model to a functioning structured finance operations unit within a major Australian bank and conclude that the model allows to include different operational loss event types.

The model proposed in this paper is based on the Advanced Measurement Approach, LDA, the OpVaR as a percentile of this distribution and the application of fuzzy logic and RMMs, integrating risk measurement and management. Accordingly, the following subsections describe the relevant conceptual foundations.

\subsection{Risk event variables}

Losses due to operational risk can be one of the following three categories [38]: expected losses (EL), unexpected losses (UL) and stress losses (SL). EL are normally of high frequency and low severity; UL are based on the EL and correspond to events with low frequency and high severity; while SL are greater than UL and derivate from low frequency events with an important severity. Thus, the variables that allow the characterization of a risk event are $[2,3,39]$ :

1. Frequency: Number of times a risk event repeats in a period of time (hour, day, week, month, year).

2. Severity: Amount of losses expressed in a currency in a period of time (hour, day, week, month, year).

Generally, frequency is modeled by using discrete distributions: Poisson or Binomial; while the severity is modeled through continuous distributions: Lognormal, Weibull or Generalized Pareto [1, 2, 39]. Given the independence between frequency and severity, not necessarily those with higher frequency they do in terms of severity, nor are those who have high frequency the low severity.

\subsection{Methodologies to determine the regulatory capital for OpRisk}

According to Basell II, organizations and financial entities have three different methods to determine the regulatory capital for OpRisk [39]:

1. Basic Indicator Approach (BIA). This indicator allows the estimation of $O p V a R_{\alpha}$ by applying a rate of $15 \%$ on financial and non-financial income for the three previous years.

2. Standardized Approach (SA). This indicator divides the activities of the financial entities in eight business lines (Corporate Finance, Trading \& Sales, Retail, Commercial, Payment \& Settlement, Agency Services, Asset Management, Retail Brokerage), each one with an associated beta factor that ranges between $12 \%$ and $15 \%$ of its corresponding generated income.

3. Advanced Measurement Approach (AMA). The aim of AMA models is to determine the aggregate LD (ALD) using the distributions of frequency and severity of risk events at a certain confidence level and time horizon. The use of these models for organizations and financial entities requires approval by their country government or financial supervisor. 


\subsection{Aggregated loss distribution}

The ALD collects historic losses by using a distribution losses matrix (DLM). The BCBS-Basel II established for the DLM the following seven risk event types associated with the business lines of an organization or financial entity: internal fraud; external fraud; labor relations; damage to fixed assets; technological damages; and execution, delivery and management of processes.

Consequently, for finance institutions, the DLM contains 56 cells corresponding to the 8 business lines and 7 event types and the operational loss will be

$$
L=\sum_{k=1}^{56} S_{k}
$$

where $S_{k}$ is the sum of losses of the $k$-th cell of the DLM. The LD approach is a method to model the random loss $S_{k}$ of a particular cell, and it assumes that $S_{k}$ is the random sum of homogeneous individual losses. To simplify we omit the subindex $k$, the loss can be written as $[2,40]$ :

$$
S=\sum_{n=1}^{N(t)} X_{n}
$$

where $N(t)$ is the random number of individual losses for the period $[0, t]$ and $X_{n}$ is the $n$-th individual loss. The LD approach is based on the following assumptions [41, 2]:

- $N(t)$ : Represents the random variable that indicates the number of events that occur in a period of time (hour, day, week, month, year). It follows the loss frequency distribution $P$ and the probability that the number of loss events is equal to $n$ is denoted by $p(n)$.

- $X$ : Random variable that indicates the quantity of losses (severity) caused by risk event in a period of time (hour, day, week, month, year). The individual losses $X_{n}$ are independent and identically distributed with loss severity distribution $F$.

- The number of events is independent from the amount of loss events.

Once the probability distributions $P$ and $F$ are chosen, the cumulative distribution function of $S, G$, can be obtained as:

$$
G(x)= \begin{cases}\sum_{n=1}^{\infty} p(n) \cdot F^{n *}(x) & x>0 \\ \sum_{n=1}^{\infty} p(n) & x=0\end{cases}
$$

where $F^{n *}$ is the $n$-fold convolution of $F$ with itself:

$$
F^{n *}(x)=\operatorname{Pr}\left[\sum_{i=1}^{n} X_{i} \leq x\right]
$$

$G(x)$ defines the LD and it is characterized by three values: expected losses (EL), unexpected loss (UL) and stress losses (as represented in Fig. 1). $O p V a R_{\alpha}$ measures the operational risk at the confidence level of $\alpha$, i.e. the level of losses that is only exceeded with a probability of $1-\alpha$ in $G(x)$. BCBS-Basel II recommends a value of $\alpha=0.001$.

The Panjer Algorithm or Montecarlo Simulation is used to obtain the distribution function $G(x)$. In this context, BCBS-Basel II recommends a database with 3 to 5 years of operational losses. However the numbers of events of this type are few, so it is important to use scenarios of risk applying Montecarlo sampling to generate random variables for frequency and severity. This recommendation is made with the possibility to incorporate new real data of risk into the model over time [40].

\subsection{Operational Value at Risk $\left(\mathrm{OpVaR} \mathrm{R}_{\alpha}\right)$}

$O p V a R_{\alpha}$ is a statistical risk measure that pretends to respond the question: How much do we can expect to lose with a certain probability over a certain period of time? Consequently, $O p V a R_{\alpha}$ is defined as the maximum loss that can be expected given a confidence level $(\alpha)$ and a time horizon (1 


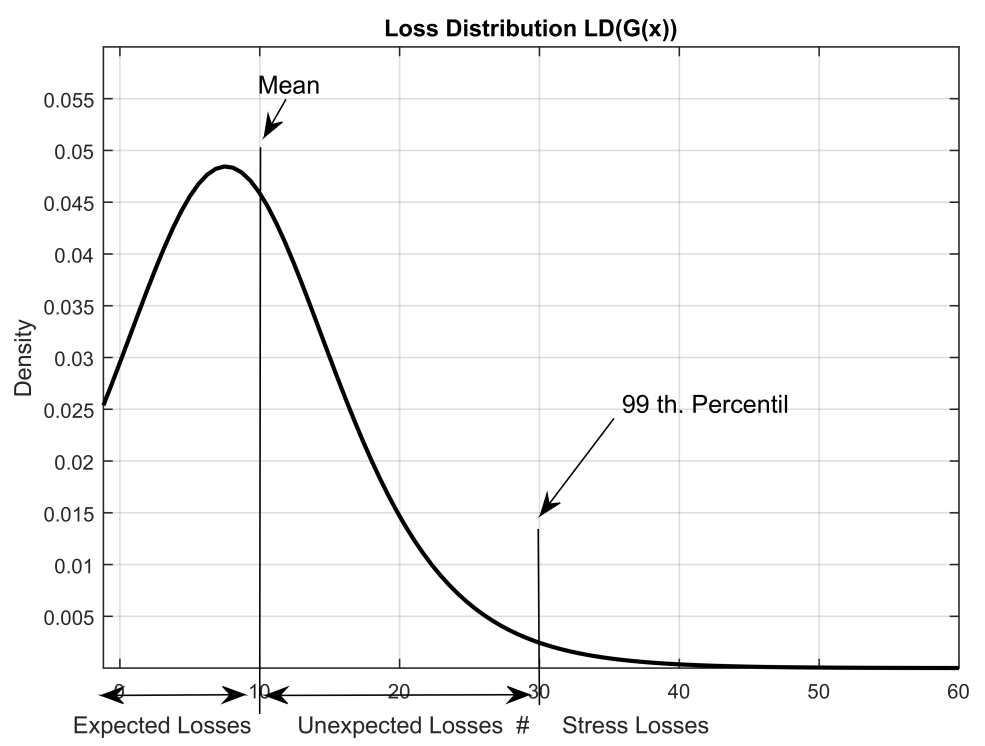

Figure 1: General structure of the loss distribution - LD $(G(x))$

year). Risk in the $j$-th event type for the $i$-th business line at confidence level of $\alpha, O p V a R(i, j, \alpha)$, is defined as follows [42]:

$$
O p \operatorname{VaR}(i, j, \alpha)=E L(i, j)+U L(i, j, \alpha)
$$

where $i$ : Line of Business $(i=1,2, \ldots, N B L) ; j$ : Type of risk event $(j=1,2, \ldots, N E R) ; N B L$ : Number of Business Lines; NER: Number of Risk Events; $E L(i, j)$ : Expected losses and it is the mean of the LDA distribution; $U L(i, j, \alpha)$ : Unexpected losses at a confidence level of $\alpha$.

The $O p V a R_{\alpha}$ aggregates the operational risk for the business lines and the operational risk events:

$$
O p V a R_{\alpha}=\sum_{i=1}^{N B L} \sum_{j=1}^{N E R} O p \operatorname{VaR}(i, j, \alpha)
$$

Three methodologies exist to estimate $O p V a R_{\alpha}$ : the historical method (simulating methodology), the parametric method and the Montecarlo method [39, 43].

\section{FIAFIm methodology}

One of the most important issues when it comes to $O p V a R_{\alpha}$ estimation is related both to working with large amounts of qualitative information that define an event of risk and the structure (type of distribution) and shape (long tail) of the LD distribution. However, the estimation of the LD by using different AMA analytical models, in many cases, does not allow to integrate RMMs to explain the evolution of risk over the time, so that the assessment of the effects of risk management on the LD can take a long time or be time consuming. For this reason, a flexible inverse adaptive fuzzy inference model (FIAFIm) is proposed in this paper aiming at explaining a priori the evolution of OpRisk over the time, using different risk profiles or RMMs.

\subsection{Experimental study design}

For the analysis and validation of the proposed model a database of 701 records of daily risk events related to the failures of cash machines in a financial entity in two years 2009-2010 (retail bank: business line) has been used. However, in order to achieve a confidence level of $99.9 \%$ for the $O p V a R_{\alpha}$, the LD of reference (LD_MC) is estimated in accordance with the guidelines given by BCBS-Basel II and, consequently, the database is extended to a total of 1000 records of risk events by applying the Monte-Carlo sampling method (see Section 3.3.2) with regard to frequency and severity [1]. This LD_MC is used by the proposed FIAFIm model as a reference in the learning phase. 


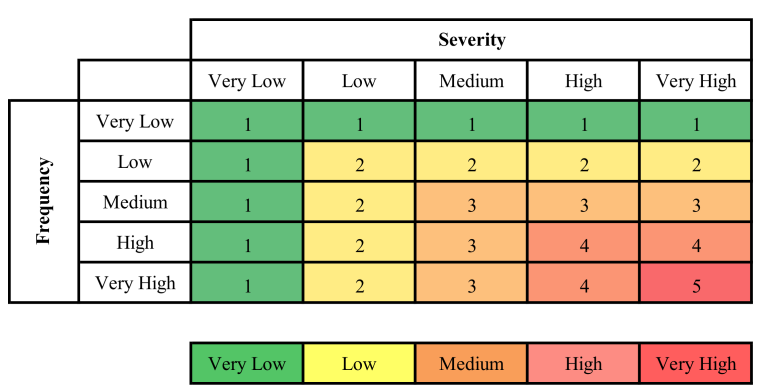

Figure 2: Risk Management Matrix (RMM).

To evaluate the behavior of the risk management evolution concerning LD a sequence of three risk profiles or RMMs was defined (E1-strong impact, E2-medium impact, E3-soft impact). Each of the RMMs is designed and defined with five rows and five columns, aligned with five qualities $(n f s)$ that define the different linguistic assessment levels for random (linguistic) input variables of frequency and severity: very low, low, medium, high, very high. The FIAFIm model wasis executed for a total of $k=500$ cycles in the learning phase, using the records of risk events from the database (1000 records). The stop criteria is defined through the difference of rms (root mean square) at instant $k+1$ and $k$ verifying $\left|r m s_{k+1}-r m s_{k}\right|<5 e-03$ for at least ten cycles of learning.

\subsection{Risk Management Matrix (RMM)}

The risk management matrix (RMM) is a structure which defines a risk profile that includes the intuition and feelings of domain experts, related to the impact and management of failures in business processes. As already mentioned, the RMM matrix requires that the input variables are represented as linguistic variables, considering both, the uncertainty in the management and the intrinsic properties that conform the random input variables for frequency and severity. The size of the matrix depends on the set of labels that describe the frequency and severity linguistic input variables [43, 44]. The colors used (see Fig. 2) show the mixed impact of frequency and severity on risk (impact matrix). The red color indicates very high impact (both frequency and impact are very high), while the dark green cells represent a very low impact (both frequency and severity are very low). The numbers indicate the management level for each impact (management matrix), where ' 1 ' indicates the lowest level of management, while ' 5 ' indicates the highest level to mitigate a mixed impact.

\subsection{Montecarlo sampling fuzzy sets}

In order to integrate the behavior of the Montecarlo sampling method into the proposed FIAFIm model, the following definitions are required:

\subsubsection{Definitions and concepts}

Definition 1. Let $X_{1}, X_{2}, \ldots ., X_{n}$ be independent and identically distributed sample from an unknown cumulative distribution function (CDF) $F(x)=P(X \leq x)$. The Empirical Distribution Function $(E C D F)$, also known simply as the empirical distribution function, is defined as [45]:

$$
F_{n}(x)=\frac{1}{n} \sum_{i=1}^{n} \mathbf{1}\left\{X_{i} \leq x\right\}
$$

where $\mathbf{1}$ is the indicator function

$$
\mathbf{1}\left\{X_{i} \leq x\right\}= \begin{cases}1 & \text { if } X_{i} \leq x \\ 0 & \text { otherwise }\end{cases}
$$

Definition 2. If CDF $F$ is strictly increasing and continuous then $F^{-1}(p)=x, p \in[0,1]$, is the unique real number $x$ such that $F(x)=p$. In such a case, this defines the inverse distribution function or quantile function. 
Definition 3. Let $\left\{q_{1}, q_{2}, \ldots ., q_{n f s}\right\}$ be the quantiles of a random variable $X$ with CDF $F(x)$. Let $\left\{X C_{N, 1}, X C_{N, 2}, \ldots, X C_{N, n f s}\right\}$ the normalized location of fuzzy sets that define the different linguistic assessment levels used for this same random variable. Let $m$ be the slope of the correlation line between the quantiles and the normalized location of fuzzy sets. Then, we have:

- When $m=1$, the fuzzy sets that define the different linguistic assessment levels for the random variable are uniformly distributed on the horizontal axis.

- When $m>1$, the fuzzy sets that define the different linguistic assessment levels for the random variable are distributed towards the left side of the horizontal axis.

- When $m<1$, the fuzzy sets that define the different linguistic assessment levels for the random variable are distributed towards the right side of the horizontal axis.

Definition 4. Given $\left\{q_{o}, q_{1}, q_{2}, q_{3}, q_{4}\right\}$ the quantiles of a random variable with CDF $F(x)$. Then we have that

$$
\frac{\partial \mathrm{F}\left(q_{2}\right)}{\partial \mathrm{x}} \approx \mathrm{m}
$$

and when (see Fig. 3):

1. $m=1: \operatorname{CDF} F(x)$ has asymmetry $=0$ and the data may come from a centered distribution (balanced fuzzy sets - Fig. 3 (a)).

2. $m>1$ : CDF $F(x)$ has asymmetry $>0$ and the data may come from a distribution with long tail (unbalanced fuzzy sets with tendency to the left side - Fig. 3 (b)).

3. $m<1$ : $\operatorname{CDF} F(x)$ has asymmetry $<0$ and data may come from a distribution with inverted long tail (unbalanced fuzzy with tendency to the right side - Fig. 3 (c)).

Compliant with Definition 4, a priori the structure and shape of the probability distribution that represent a linguistic variable can be set.

\subsubsection{Montecarlo fuzzy sampling method}

Let $\left\{q_{1}, q_{2}, \ldots . ., q_{n f s}\right\}$ be the quantiles of a random variable $X$ with $\operatorname{CDF} F(x)$, which is also described linguistically via $n f s$ fuzzy linguistic labels with following Gaussian membership functions:

$$
u_{j}(x)=\operatorname{Exp}\left(-\frac{1}{2}\left(\frac{q_{j}-x}{D_{j}}\right)^{2}\right)
$$

where

$$
D_{j}= \begin{cases}q_{j+1}-q_{j-1} & j<n f s \\ 2 \cdot\left(q_{j}-q_{j-1}\right) & j=n f s \\ 2 \cdot\left(q_{j+1}-q_{j}\right) & j=0\end{cases}
$$

The Monte-Carlo fuzzy sampling function for a uniform random value $p_{k} \in[0,1], F S\left(p_{k}\right)$, has the following structure [46]:

$$
F S\left(p_{k}\right)=\sum_{j=1}^{n f s} u_{j}\left(p_{k}\right) \cdot F^{-1}\left(q_{j}\right)
$$

where $F^{-1}\left(q_{j}\right)$ is the quantile function for $q_{j}$. Thus, the procedure of sampling by using the MonteCarlo method for a random variable $X$ with CDF $F(x)$ described linguistically via a number of fuzzy linguistic sets is:

1. Normalize fuzzy linguistic sets used to describe the random variable.

2. Generate random number $p_{k} \in[0,1]$ using a uniform distribution.

3. Compute degrees of membership of random generated value in step 2 with respect to the normalized fuzzy sets $j$ using $(4), u_{j}\left(p_{k}\right)$.

4. The sampling value is obtained using (6).

5. Steps 1, 2 and 3 are repeated until 1000 data is reached. 

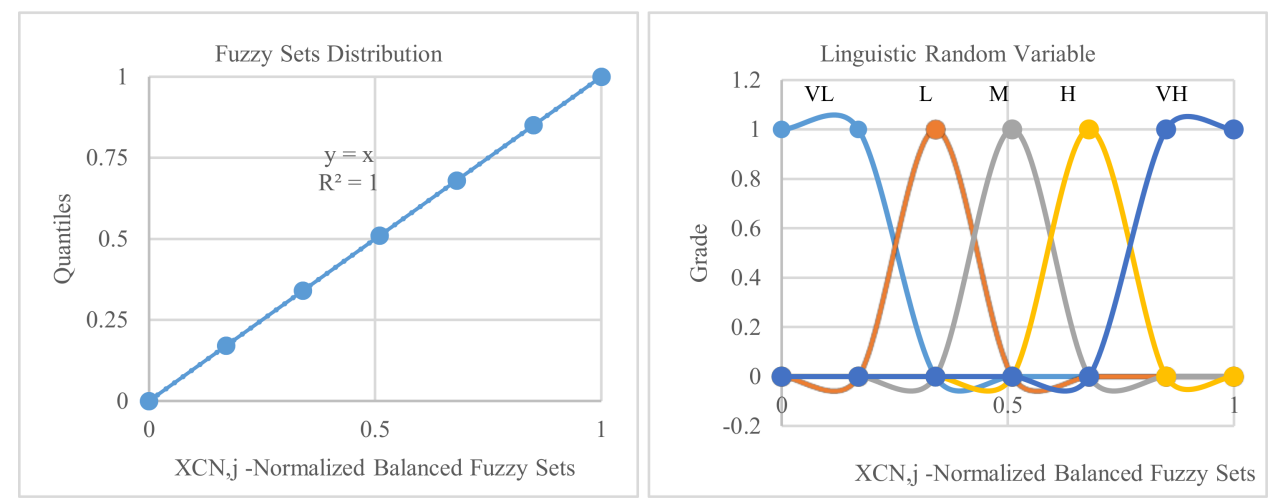

(a)

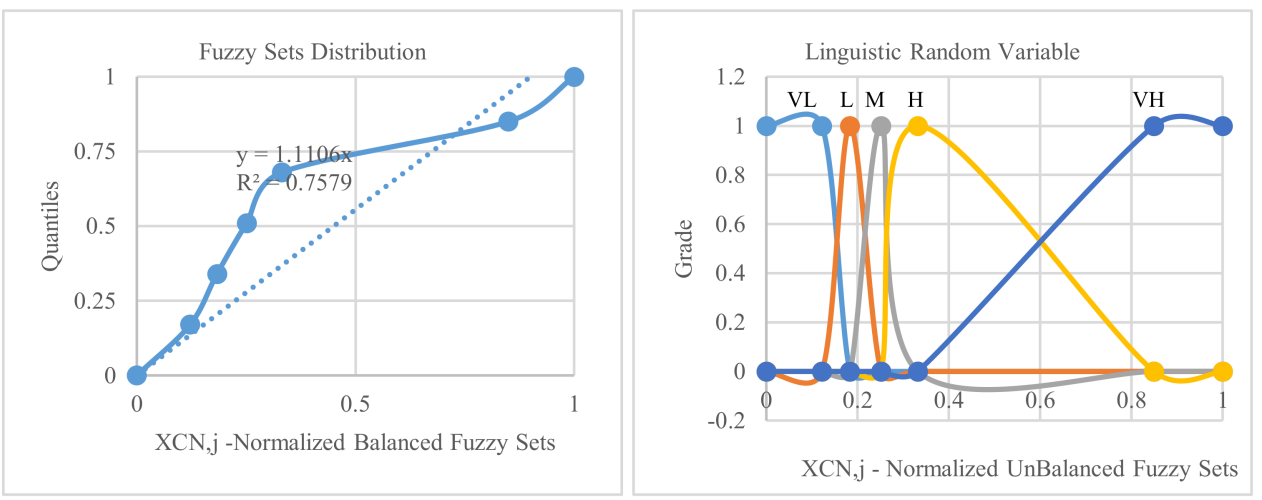

(b)

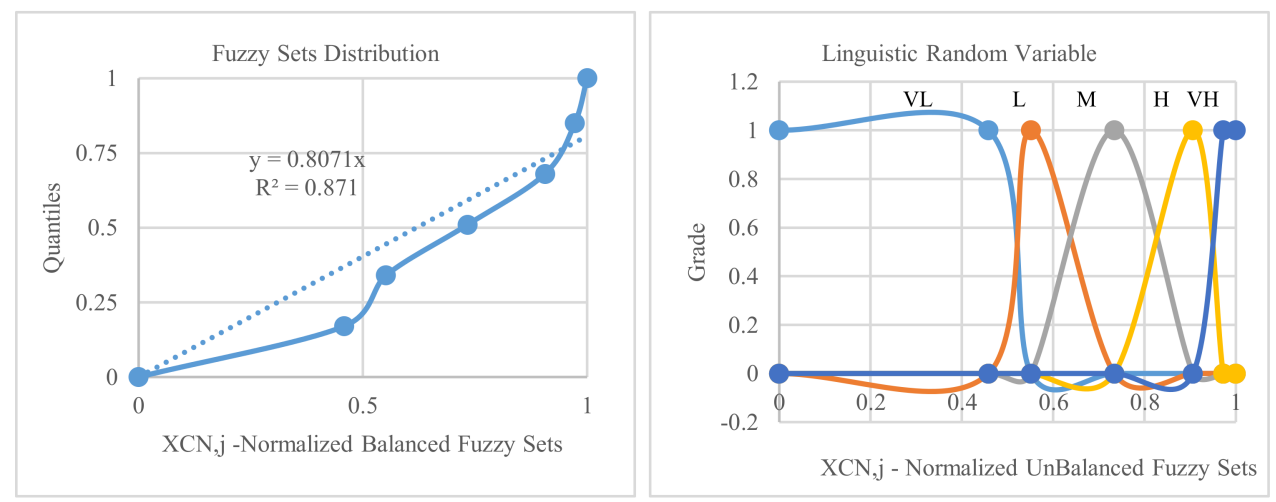

(c)

Figure 3: Location of fuzzy sets: (a) when $m=1$, the fuzzy sets are located uniformly in [0,1], (b) when $m>1$, the fuzzy sets are located toward the left side of $[0,1],(\mathrm{c})$ when $m<1$, the fuzzy sets are located toward the right side of $[0,1]$. VL: Very Low, L: Low, M: Medium, H: High, VH:Very High 


\subsection{Flexible Adaptive Fuzzy Inference Model with Montecarlo Structure (FIAFIm) to assess the Fuzzy Op VaR}

\subsubsection{Flexible Adaptive Fuzzy Inference Model with Montecarlo Structure (FIAFIm)}

To estimate the LD, a FIAFIm model using a fuzzy inference model derived from the aforementioned RMMs is proposed, each one with an associated value of losses that is estimated by applying a Montecarlo sampling method as described below:

$$
\begin{gathered}
\text { if } x_{f, k} \text { is } X C_{j, f}^{l} \text { and } x_{s, k} \text { is } X C_{j, s}^{l} \text { then } w_{k}^{l} \text { is } B_{j}^{l}: \\
y_{k}^{l}=C_{k}^{l} \cdot L R_{k}^{l} \cdot M P^{l} \cdot x_{f, k} \cdot x_{s, k}
\end{gathered}
$$

being $i$ representing the $i$-input random linguistic variable ( $f$ (frequency), s(severity)); $j$ the $j$-fuzzy set defined for each of the following linguistic variable $(n f s)$ : very low: 1, low: 2, medium: 3 , high: 4 , very high: $5 ; l$ the system's rules $(l=1,2, \ldots, n f s \times n f s) ; x_{f, k}$ the frequency sampling value at instant $k$ (number of training data); $x_{s, k}$ the severity sampling value at instant $k ; X C_{j, i}^{l}$ the centroid of $j$-fuzzy set for the input $i$-variable $(i=f, s)$ of rule $l ; w_{k}^{l}$ the consequent of the rule $l$ calculates as

$$
\begin{gathered}
w_{k}^{l}=u f_{j 1, k} \cdot u s_{j 2, k} \\
j_{1}=1,2, \ldots, n f s ; j_{2}=1,2, \ldots, n f s
\end{gathered}
$$

with $u f_{k}$ being the frequency membership degree associated to $\left(x_{f, k}\right.$ is $\left.X C_{j, f}\right)$; us $s_{k}$ the severity membership value associated to $\left(x_{s, k}\right.$ is $\left.X C_{j, s}\right) ; B_{j}{ }^{l}$ is the balanced fuzzy sets that represent the level of risk management associated with the corresponding $j$-levels that define the risk in rule $l$ : very low: 1, low: 2, medium: 3, high: 4 , very high: 5 (Fig. 4 ); $L R_{k}^{l}$ the level of risk management given by the $l$-rule (at instant $k$ )

$$
L R_{k}^{l}=w_{k}^{l} \cdot B^{l}
$$

with $C_{k}^{l}$ indicating the level of management of the $l$-rule; $M P^{l}$ the RMM risk profile associated to rule $l$; and $y_{k}^{l}$ the value of losses estimated by rule $l$ at instant $k$, which will shape the LD experimental distribution or loss distribution approach (LDA).

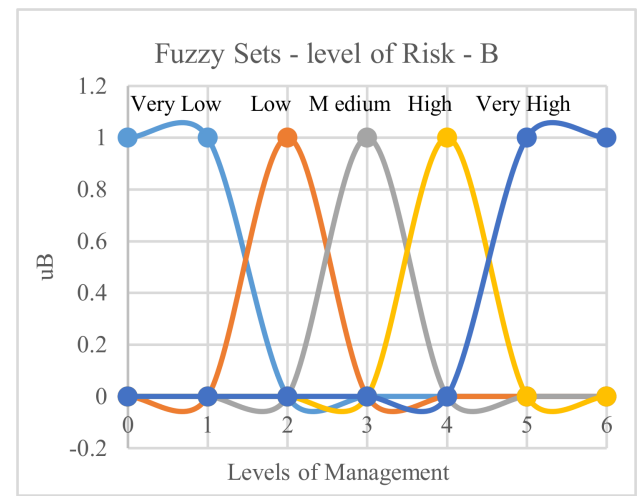

Figure 4: Output - fuzzy sets that represent the level of risk

The final output value of the FIAFIm is obtained as follows:

$$
\begin{gathered}
y r_{L D A_{k}}=\sum_{l=1}^{N R} y_{k}^{l} / S M D \\
S M D=\sum_{l=1}^{N R} M P^{l}
\end{gathered}
$$

where $N R$ is the number of rules that conform the fuzzy system. 
The adaptive process is defined by the generalized delta rule [47]:

$$
\begin{gathered}
C_{k+1}^{l}=C_{k}^{l}-\gamma \cdot \frac{\partial e_{k}^{2}}{\partial C_{k}^{l}} \\
X C^{l}{ }_{j, i, k+1}=X C_{j, i, k}^{l}-\gamma \cdot \frac{\partial e_{k}^{2}}{\partial X C^{l}{ }_{j, i, k}} \\
D_{j, i, k+1}^{l}=D_{j, i, k}^{l}-\gamma \cdot \frac{\partial e_{k}^{2}}{\partial D^{l}{ }_{j, i, k}}
\end{gathered}
$$

where $D_{j, i}, k$ is the size of the base of the $j$-fuzzy set of the $i$-input variable at instant - $k$ (see expression (5)); $\gamma$ represents the learning factor; $e_{k}^{2}$ is the rot mean square error (rse) at instant $k$ :

$$
e_{k}^{2}=\left(y d_{k}-y r_{L D A, k}\right)^{2}
$$

and $y d_{k}$ is the LD_MC obtained by Montecarlo sampling according to Basel II_BCBS. This way the learning rules take the following form:

$$
\begin{gathered}
C_{k+1}^{l}=C_{k}^{l}+2 \cdot \gamma \cdot e_{k} \cdot\left(\frac{M P^{l}}{S M D}\right) \cdot L R_{k}^{l} \cdot x_{s, k} \cdot x_{f, k} \\
X C_{j, i, k+1}^{l}=X C_{j, i, k}^{l}+2 \cdot \gamma \cdot e_{k} \cdot\left(\frac{C^{l} M P^{l}}{S M D}\right) \cdot x_{s, k} \cdot x_{f, k} \cdot B^{l} \cdot w^{l} \cdot\left(\frac{x_{i, k}-X C_{j, i}}{D_{j, i}^{l 2}}\right) \\
D_{j, i, k+1}^{l}=D_{j, i, k}^{l}+2 \cdot \gamma \cdot e_{k} \cdot\left(\frac{C^{l} M P^{l}}{S M D}\right) \cdot x_{s, k} \cdot x_{f, k} \cdot B^{l} \cdot w^{l} \cdot\left(\frac{x_{i, k}-X C_{j, i}}{D^{l}{ }_{j}{ }^{3}}\right)
\end{gathered}
$$

As discussed in previous sections, the FIAFIm model can take three different forms to estimate the LD_MC in the learning phase:

1. Inverse Adaptive Uniform Fuzzy Inference Model (IAUFIm) to identify the RMM - TLM $M^{l}$ using balanced fuzzy sets and equation (14) (Setting RMM).

2. Inverse Adaptive Non Uniform Fuzzy Model (IANUFIm) to identify the RMM - TLM $M^{l}$ using adaptive unbalanced fuzzy sets and equations (14), (15) and (16) (Setting the fuzzy input sets).

3. Inverse Adaptive Sampling Fuzzy Inference Model (IASFIm) to identify the RMM - TLM $M^{l}$ using fuzzy sets obtained through the Montecarlo sampling method and equations (6), (14), (15) and (16) (Evolution of risk).

After the learning process, the setup model gives, in terms of a result, the following true risk management matrix:

$$
T L M^{l}=C^{l} \cdot B^{l} \cdot M P^{l}
$$

where $T L M^{l}$ is the true level management vector representing the true risk management in an organization with the aim to mitigate the effects generated by its OpRisk. This vector can be converted in an array with the aim to compare this array with the array that shows the impact of the risk. The main diagonal of $T L M^{l}$ allows building a linguistic variable that determines the true levels of risk management that is achieved by the model after the learning process. Notice that changing $M P^{l}$ will allow to integrate in the FIAFIm model different risk profiles or RMMs, with the aim to assess its impact on the LDA distribution.

\subsubsection{Fuzzy $O p V a R$}

Let $F(x)$ be the cumulative distribution function (CDF) of a random variable $X$, and $u$ the value of $X$ in the tail of the distribution, then the probability of $X$ being located between $u$ and $u+y(y>0)$ when $x>u$ is:

$$
P[u<X<u+y \mid x>u]=\frac{P[u<x<u+y]}{P[x>u]}=\frac{F(u+y)-F(u)}{1-F(u)}=F_{u}(y)
$$


$F_{u}(y)$ represents the right tail of the distribution of probability. According to Gnedenko et al. [48], for a wide class of distributions $F(x)$, increasing the $u$ variable leads to $F_{u}(y)$ to converge to the following generalized Pareto distribution (GPD):

$$
G_{\xi, \beta}(y)=1-\left(1+\frac{\xi \cdot(y-u)}{\beta}\right)^{-\frac{1}{\xi}}, \xi \neq 0
$$

where $u$ is a threshold parameter; $\beta$ is a scale parameter $(\beta>0)$; and $\xi$ is a shape parameter $(\xi \epsilon R)$.

The estimation of $1-F(u)$ can be determined from the empirical data:

$$
\begin{gathered}
1-F(u)=P[x>u]=\frac{n_{u}}{n} \\
P[x>u+y]=P[x>u+y \mid x>u] \cdot P[x>u]=\frac{n_{u}}{n} \cdot\left[1-G_{\xi, \beta}(y)\right]
\end{gathered}
$$

where $u$ representing a value close to the $95 \%$ percentile of the empirical distribution $(u \in R) ; n$ is the total number of observations that make up the empirical distribution; and $n_{u}$ is the number of observations of $x$ that are greater than $u$. Therefore, the estimator of the tail of the CDF $F(x)$, when $x$ is big, can be expressed as follows:

$$
F(x)=P[x<u+y]=1-P[x>u+y]=1-\frac{n_{u}}{n} \cdot\left(1+\xi \cdot \frac{(x-u)}{\beta}\right)^{-1 / \xi}
$$

According to (2), in order to calculate the $O p \operatorname{VaR}$ with a confidence level of $q$ it is necessary to solve the following equation:

$$
q=1-\frac{n_{u}}{n} \cdot\left(1+\xi \cdot \frac{(O p V a R-u)}{\beta}\right)^{-1 / \xi}
$$

Thus, we have that $O p V a R$ is:

$$
O p V a R=u+\frac{\beta}{\xi} \cdot\left[\left(\frac{n_{u}}{n} \cdot \frac{1}{(1-q)}\right)^{\xi}-1\right]
$$

In line with the fuzzy representation of the LD distribution from Definition 3 , the parameters $\xi$ and $\beta$ can be estimated from:

$$
q_{j}=1-\frac{n_{u}}{n} \cdot\left(1+\xi \cdot \frac{L D^{-1}\left(q_{j}\right)-u}{\beta}\right)^{-1 / \xi}
$$

where $L D^{-1}\left(q_{j}\right)$ is the quantile function that represents the CDF of the LD distribution for the value $q_{j}$ that are the different values according to the number of fuzzy sets that define the random variable of interest. In the particular case of using $j=1$ (Very Low), 2 (Low), 3 (Medium), 4 (High), 5 (Very High), the quantiles for the LD experimental distribution are defined as $q_{0}, q_{0.25}, q_{0.5}, q_{0.75}, q_{1}$, respectively for the experimental LD given by the fuzzy model.

\subsubsection{Case study}

In coherence with the structure defined by the FIAFIm, the behavior of the IUAFIm is described through the estimation of LD_MC in the learning phase:

1. It begins with the normalization of data for frequency $\left(X_{f}\right)$ and severity $\left(X_{s}\right)$, and with estimating the LD_MC of reference $\left(y d_{k}\right)$ using the Montecarlo method described in Section 3.3.2.

2. It defines the input (Freq.: $X_{N, f}$, Sev.: $X_{N, s}$ ) and output (level of management: $B$ ) linguistic variables, using balanced fuzzy sets with equal number of labels (1: very low, 2: low, 3: medium, 4: high, 5: very high). In line with the Definition 3, the balanced fuzzy sets have slope values (m) close to one, as represented in Fig. 5 (b). 


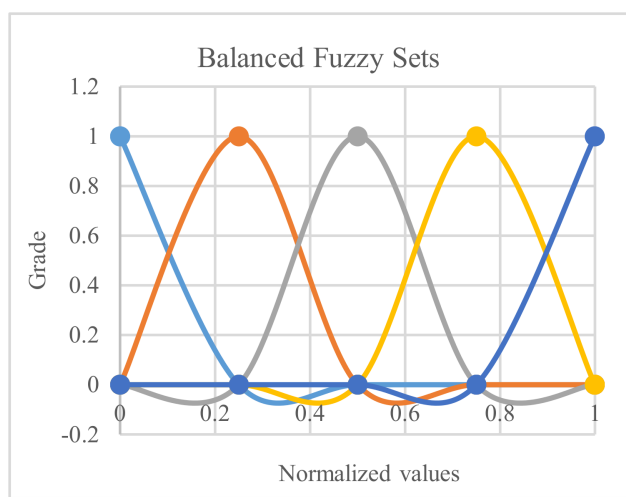

(a)

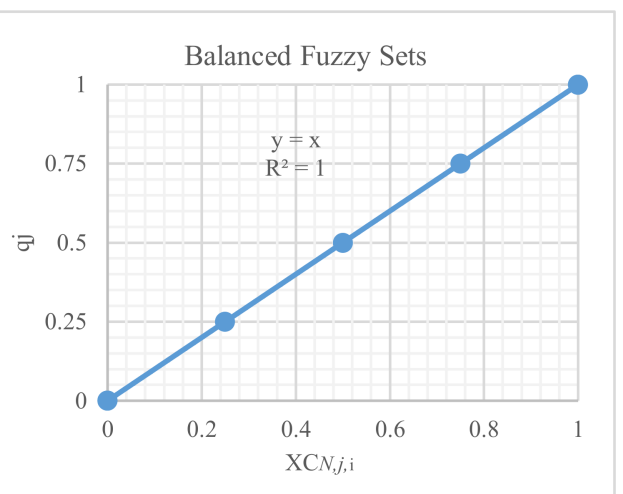

(b)

\begin{tabular}{|c|c|c|c|c|c|}
\hline & Very Low & Low & Medium & High & Very High \\
\hline$X C_{N, j, i}$ & 0 & 0.25 & 0.5 & 0.75 & 1 \\
\hline$D_{j, i}$ & 0.5 & 0.5 & 0.5 & 0.5 & 0.5 \\
\hline$B_{j, i}$ & 1 & 2 & 3 & 4 & 5 \\
\hline
\end{tabular}

(c)

Figure 5: Structure of the linguistic variables that represent the input and output variables for the IUAFm (a) Balanced fuzzy sets (BFS) (b) slope of BFS $(\mathrm{m}=1)$ (c) Location and shape of the FS

3. To estimate the LD_MC in the learning phase, the IAUFIm model uses the following neutral vector $\left(M P^{l}\right)$ for the labels that define the linguistic input variables:

$$
M P_{n f s \times n f s}=\left(\begin{array}{ccccc}
1 & 1 & 1 & 1 & 1 \\
1 & 1 & 1 & 1 & 1 \\
1 & 1 & 1 & 1 & 1 \\
1 & 1 & 1 & 1 & 1 \\
1 & 1 & 1 & 1 & 1
\end{array}\right)
$$

After the learning process this matrix may be changed by different RMMs (E1-E2-E3) with the aim to assess the impact of management on LD.

4. It proceeds with the vector setup $C^{l} \in[0,1]$, which may take the same structure as the $M P_{n f s \times n f s}$ array $\left(C_{n f s \times n f s}\right)$. This vector shows the level of management by the $l$-rule.

5. At this point the learning phase begins. Let $x_{f, k}=0.35$ and $x_{s, k}=0.71$ be two random values for frequency and severity respectively at instant $k$. Membership values are calculated (Table 1 ) using equation (4):

$$
u_{i, j, k}\left(x_{i, k}\right)=\operatorname{Exp}\left(-\frac{1}{2}\left(\frac{X C_{j, i, k}-x_{i, k}}{D_{j, i, k}}\right)^{2}\right)
$$

where $i$ represents the linguistic input variables $(i=f, s)$, and $j$ the fuzzy sets that make up the input variables ( $j_{1}:$ Freq. Variable; $j_{2}:$ Sev. Variable).

$$
\begin{aligned}
& u_{f_{2, k}}(0.35)=\operatorname{Exp}\left(-\frac{1}{2}\left(\frac{0.35-0.25}{0.5}\right)^{2}\right)=0.9801 \\
& u_{f_{3, k}}(0.35)=\operatorname{Exp}\left(-\frac{1}{2}\left(\frac{0.35-0.5}{0.5}\right)^{2}\right)=0.9559
\end{aligned}
$$


Table 1: Estimation of membership values for input values for $x_{f, k}$ and $x_{s, k}$

\begin{tabular}{lccccc}
\hline & Very Low & Low & Medium & High & Very High \\
\hline$X C_{j, f}$ & 0 & 0.25 & 0.5 & 0.75 & 1 \\
\hline$X C_{j, s}$ & 0 & 0.25 & 0.5 & 0.75 & 1 \\
\hline$D_{j, i}$ & 0.5 & 0.5 & 0.5 & 0.5 & 0.5 \\
\hline$u_{f, j 1, k}$ & 0 & 0.9801 & 0.9559 & 0 & 0 \\
\hline$u_{s, k}$ & 0 & 0 & 0.9155 & 0.9968 & 0 \\
\hline
\end{tabular}

6. Linked to the aforementioned RMM of Figure 2 the activation rules take the following values (Figure 6):

$\left\{B^{8}(\right.$ Low : 2$), B^{9}\left(\right.$ Low : 2), $B^{13}($ Medium $: 3), B^{14}($ Medium : 3$\left.)\right\}$

\begin{tabular}{|c|c|c|c|c|c|c|}
\cline { 2 - 7 } \multicolumn{1}{c|}{} & \multicolumn{5}{c|}{ Severity } \\
\cline { 2 - 8 } \multicolumn{1}{c|}{} & Very Low & Low & Medium & High & Very High \\
\hline \multirow{3}{*}{} & Very Low & & & & & \\
\cline { 2 - 7 } & Low & & & Low & Low & \\
\cline { 2 - 7 } & Medium & & & Medium & Medium & \\
\cline { 2 - 7 } & High & & & & & \\
\cline { 2 - 7 } & Very High & & & & & \\
\hline
\end{tabular}

Figure 6: Matrix of activation rules linked to the labels that describe the input linguistic variables

7. Table 2 shows the estimated output $\left(y r_{L D A, k}\right)$ produced by the IAUFm.

Table 2: Estimation of $y r_{L D A, k}$ by using the IAUFm $y r_{L D A, k}$

\begin{tabular}{|c|c|c|c|}
\hline$x_{f, k}$ & $x_{f, k}$ & $x_{f, k}$ & $x_{f, k}$ \\
\hline 0.35 & 0.35 & 0.35 & 0.35 \\
\hline$x_{s, k}$ & $x_{s, k}$ & $x_{s, k}$ & $x_{s, k}$ \\
\hline 0.71 & 0.71 & 0.71 & 0.71 \\
\hline$w^{8}=u f_{2, k} \cdot u s_{3, k}$ & $w^{9}=u f_{2, k} \cdot u s_{4, k}$ & $w^{13}=u f_{3, k} \cdot u s_{3, k}$ & $w^{14}=u f_{3, k} \cdot u s_{4, k}$ \\
\hline 0.8972 & 0.9769 & 0.8751 & 0.9528 \\
\hline$B^{8}$ & $B^{9}$ & $B^{13}$ & $B^{14}$ \\
\hline 2 & 2 & 3 & 3 \\
\hline$L R^{8}$ & $L R^{9}$ & $L R^{13}$ & $L R^{14}$ \\
\hline 1.7944 & 1.9538 & 2.6253 & 2.8584 \\
\hline$C^{8}$ & $C^{9}$ & $C^{13}$ & $C^{14}$ \\
\hline 0.49838 & 0.3033 & 0.0566 & 0.8761 \\
\hline$M P^{8}$ & $M P^{9}$ & $M P^{13}$ & $M P^{14}$ \\
\hline 1 & 1 & 1 & 1 \\
\hline \multicolumn{4}{|c|}{$S M D=M P^{8}+M P^{9}+M P^{13}+M P^{14}=4$} \\
\hline$y r^{8}$ & $y r^{9}$ & $y r^{13}$ & $y r^{14}$ \\
\hline 0.0555 & 0.0368 & 0.0092 & 0.1555 \\
\hline$\gamma$ & & \multicolumn{2}{|c|}{0.1} \\
\hline$y r_{L D A}$ & & \multicolumn{2}{|l|}{0.2570} \\
\hline$y d_{k}$ & & \multicolumn{2}{|l|}{0.3512} \\
\hline$e r_{k}$ & & \multicolumn{2}{|l|}{0.0942} \\
\hline
\end{tabular}


8. To update the structure of the IAUFIm for the 9-rule:

$$
\begin{aligned}
& C_{k+1}^{9}=C_{k}^{9}+2 \cdot \gamma \cdot e_{k} \cdot\left(\frac{M P^{9}}{S M D}\right) \cdot L R_{k}^{9} \cdot x_{s, k} \cdot x_{f, k} \\
& C_{k+1}^{9}=0.3033+2 \times 0.1 \times 0.0942 \times\left(\frac{1}{4}\right) \times 1.9538 \times 0.71 \times 0.35
\end{aligned}
$$

In addition to the update of the vector $C_{k}^{l}$, the variable $X C_{j, i, k}^{l}$ can also be updated by specifying fuzzy sets that are the target of an update. For the 9-rule the update $X C_{j, i, k}^{l}$ has the following procedure:

$$
\begin{gathered}
w^{9}=u f_{2, k} \cdot u s_{4, k} \\
X C_{2, f, k+1}^{9}=X C_{2, f, k}^{9}+2 \cdot \gamma \cdot e_{k} \cdot\left(\frac{C k^{9} M P^{9}}{S M D}\right) \cdot x_{s, k} \cdot x_{f, k} \cdot B^{9} \cdot w^{9} \cdot\left(\frac{x_{f, k}-X C_{2, f}}{D_{2, f}^{2}}\right) \\
X C_{2, f, k+1}^{9}=0.25+2 \times 0.1 \times 0.0942 \times\left(\frac{0.3033 \times 1}{4}\right) \times 0.71 \times 0.35 \times 2 \times 0.9769 \times\left(\frac{0.5-0.35}{0.5^{2}}\right) \\
X C_{4, s, k+1}^{9}=X C_{4, s, k}^{9}+2 \cdot \gamma \cdot e_{k} \cdot\left(\frac{C k^{9} M P^{9}}{S M D}\right) \cdot x_{s, k} \cdot x_{f, k} \cdot B^{9} \cdot w^{9} \cdot\left(\frac{x_{s, k}-X C_{j, s}}{D_{j, s}^{2}}\right) \\
X C_{4, s, k+1}^{9}=0.5+2 \times 0.1 \times 0.0942 \times\left(\frac{0.3033 \times 1}{4}\right) \times 0.71 \times 0.35 \times 2 \times 0.9769 \times\left(\frac{0.71-0.75}{0.5^{2}}\right)
\end{gathered}
$$

This procedure is similar to updating the diameter $D_{j, i}^{l}$ of the fuzzy sets that conform the linguistic input variables.

9. This estimation process continues until $k=1000$. When $k \leq 1000$, the process moves to step 5 .

10. This process (steps $5-9$ ) is repeated until a number of iterations (NI) is reached or up to the limit of $\left|e r s_{i 1+1}-e r s_{i 1}\right| e r s<5 e-03$ for ten consecutively iterations. When these conditions are not met, the process jumps to step 5 .

Figure 7 shows that the IUAFm finished with the IOA (Index of agreement) close to one with regard to the estimation of LDA_MC by using a neutral matrix for $M P^{l}$. That shows the good performance of the model in the learning phase.

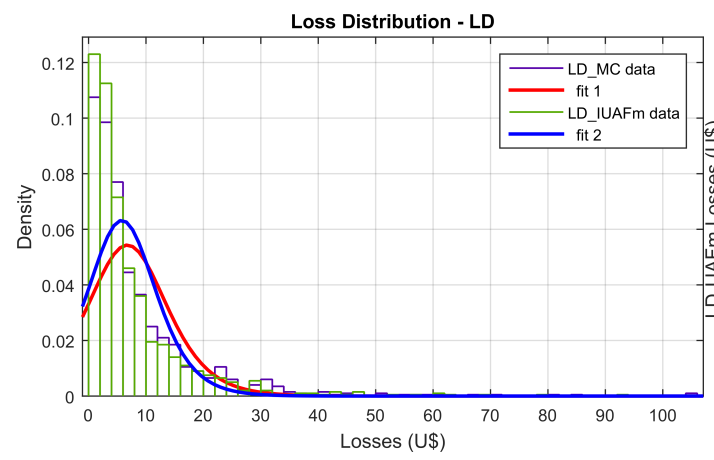

(a)

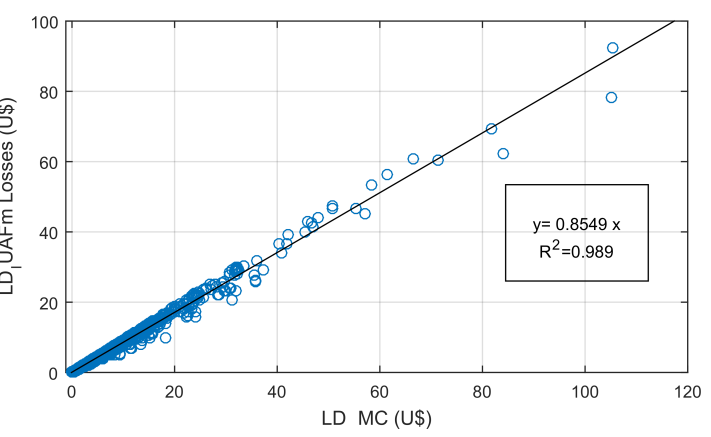

(b)

Figure 7: LD estimated by the IAUFm in the learning phase (a) pdf function (b) IOA chart 


\subsection{Experimental validation of the FIAIFm model}

To evaluate the behavior of the FIAFIm model, two stages were taken into account:

1. Learning phase stage. At this stage, the analysis of the model was carried out using the index of agreement (IOA) produced by the model (IAUFIm, IANUFIm, IASFIm) in the estimation of the LD_MC from a neutral RMM - $M P^{l}$, and the database that defines the random input variables for frequency and severity. The expectation at this stage is that the input fuzzy sets are located towards lower frequencies $\left(m_{f} \geq 1\right)$ and lower losses $\left(m_{s} \geq 1\right)$ as per Definitions 3 and 4 , except for the IAUFIm, which uses input balanced fuzzy sets $\left(m_{f}=m_{s}=1\right)$.

2. Evolution process stage. At this stage, and after running the learning phase stage, the analysis of the model was executed by using a sequence of risk profiles (RMMs) (E1-strong impact, E2medium impact, E3-soft impact), which shows the natural evolution of risk management in an organization as per Fig. 8, where the RMMs with smaller values represent smaller impacts on LD due to the effect of a better risk management.

\begin{tabular}{|c|c|c|c|c|c|}
\hline & 0 & 1 & 2 & 3 & 4 \\
\hline 0 & 2 & 4 & 8 & 12 & 16 \\
\hline 1 & 4 & 4 & 8 & 12 & 16 \\
\hline 2 & 8 & 8 & 8 & 12 & 16 \\
\hline 3 & 12 & 12 & 12 & 12 & 16 \\
\hline 4 & 16 & 16 & 16 & 16 & 16 \\
\hline
\end{tabular}

(a)

\begin{tabular}{|l|l|l|l|l|l|}
\hline & 0 & 1 & 2 & 3 & 4 \\
\hline 0 & 2 & 2 & 2 & 4 & 6 \\
\hline 1 & 2 & 2 & 2 & 4 & 6 \\
\hline 2 & 2 & 4 & 4 & 4 & 6 \\
\hline 3 & 4 & 4 & 6 & 6 & 8 \\
\hline 4 & 4 & 6 & 6 & 8 & 8 \\
\hline
\end{tabular}

(b)

\begin{tabular}{|l|l|l|l|l|l|}
\hline & 0 & 1 & 2 & 3 & 4 \\
\hline 0 & 0 & 0 & 0 & 0 & 0 \\
\hline 1 & 0 & 1 & 1 & 1 & 1 \\
\hline 2 & 0 & 1 & 2 & 2 & 2 \\
\hline 3 & 0 & 1 & 2 & 3 & 3 \\
\hline 4 & 0 & 1 & 2 & 3 & 4 \\
\hline
\end{tabular}

(c)

Figure 8: Sequence of evolution of risk management in an organization: (a) E3-Soft Impact (b) E2-Medium Impact (c) E1-Strong Impact

In order to assess the impact that risk management generates on the LD, an IC-fingerprint structure is generated by grouping the main statistical indices that characterize an $\operatorname{LD}[49,28]$ :

1. $O p V a R_{99.9 \%}$ : Operational risk value set at the 99.9 percentile on the LD distribution.

2. EL (Expected Losses): Indicates the expected losses. This value is represented by the median of the LD distribution.

3. UL (Unexpected losses): Indicates the unexpected losses. This values is defined as:

$$
U L=O p V a R_{99.9 \%}-E L
$$

4. Coverage of EL (CEL): Indicates the percentage of coverage of EL by the $O p V a R_{99.9 \%}$.

$$
C E L=\frac{E L}{O p V a R_{99.9 \%}}
$$

5. Coverage of UL (CUL): Indicates the percentage of coverage of UL by the $O p V a R_{99.9 \%}$.

$$
C U L=\frac{U L}{O p V a R_{99.9 \%}}
$$

6. Tail data (TD): Number of data located in the tail of the LD distribution, i.e. between the $E L$ and $O p V a R_{99.9 \%}$ values. 
7. Exposure Grade (EG): Percentage of coverage reached by $O p V a R_{99.9 \%}$ with regard to the average losses located in the tail of the LD distribution.

$$
E G=\frac{\left(\frac{T D}{N D}\right) \cdot\left(\frac{O p V a R_{99.9 \%}+U L}{2}\right)}{O p V a R_{99.9 \%}}
$$

8. Insured value (IV): Indicates the insured value to cover the events of risk at the level of $99.9 \%$.

$$
I V=O p V a R_{99.9 \%} E G
$$

After the learning process, and in line with the behavior of the IAUFIm, the evolution experienced by the LD as a result of better risk management that defines the sequence of risk (E1-E2-E3) can be observed in Table 3 and Fig. 9. The LD evolve toward lower values of scale $(\sigma), O p V a R_{99.9 \%}$, EL, UL and insurance, getting close to the LD_MC, as defined by the BCBS-Basel II, which represents standards for OpRisk management [1].

Table 3: Impact of risk management on the LD distribution

\begin{tabular}{lccccc}
\hline & LDA_MC & LDA_IAUfm & LDA_E1 & LDA_E2 & LDA_E3 \\
\hline Distribution & G.Pareto & G.Pareto & G.Pareto & G.Pareto & G.Pareto \\
\hline AIC & -3708.41 & -3646.83 & -4290.88 & -5342.16 & -5961.9 \\
\hline shape $(k)$ & 0.1755 & 0.1755 & 0.1879 & 0.1847 & 0.1824 \\
\hline scale $(\sigma)$ & 7.1078 & 6.1207 & 13.1796 & 37.4881 & 68.1123 \\
\hline threshold $(\theta)$ & $-2.2204 \mathrm{E}-15$ & $-2.2204 \mathrm{E}-15$ & $-2.2204 \mathrm{E}-15$ & $-2.2204 \mathrm{E}-15$ & $-2.2204 \mathrm{E}-15$ \\
\hline kurtosis & 20.31103546 & 17.94675136 & 19.46681829 & 19.01564618 & 18.71939461 \\
\hline assymetry & 3.60797988 & 3.443027288 & 3.576584394 & 3.537899709 & 3.512063762 \\
\hline OpVaR $99.9 \%$ & 105.1600463 & 78.23280913 & 179.4159257 & 503.299556 & 904.1246106 \\
\hline EL & 8.635985603 & 7.434086364 & 16.25420563 & 46.0520088 & 83.43525422 \\
\hline UL & 96.52406075 & 70.79872277 & 163.16172 & 457.2475472 & 820.6893563 \\
\hline CEL & 0.082122307 & 0.095025175 & 0.090595111 & 0.091500198 & 0.092282915 \\
\hline CUL & 0.917877693 & 0.904974825 & 0.909404889 & 0.908499802 & 0.907717085 \\
\hline TD & 321 & 320 & 317 & 317 & 318 \\
\hline EG & 0.30781937 & 0.304795972 & 0.302640675 & 0.302497219 & 0.303327017 \\
\hline IV & 3.237029919 & 2.38450451 & 5.429855684 & 15.22467159 & 27.42454207 \\
\hline IOA & 1 & 0.994528444 & 0.995859429 & 0.995786996 & 0.99560061 \\
\hline & & & & & \\
\hline
\end{tabular}

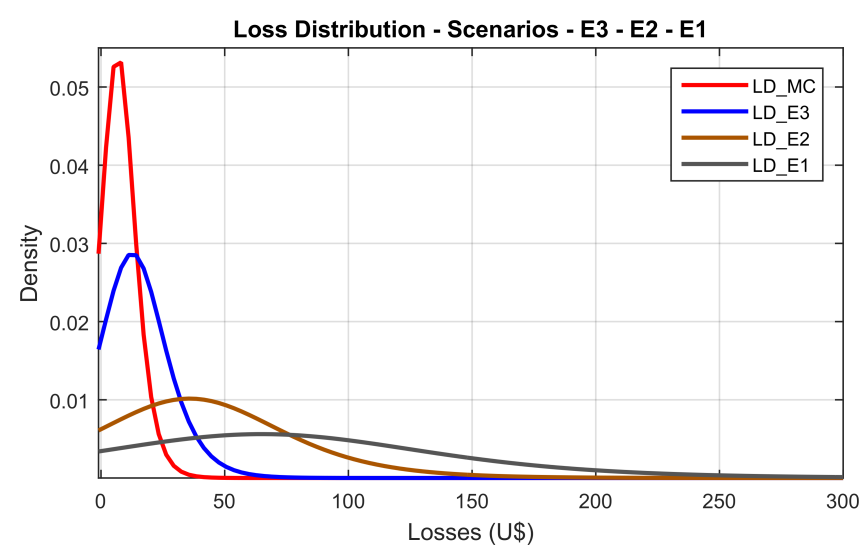

Figure 9: LD estimated by the model according to the sequence of risk management 
Indeed, the experimental validation is mainly based on the following: In order to evaluate the evolution of the aggregate loss distribution for different management scenarios (states), the estimation of the LD is carried out first, using a risk management matrix and impact with similar values of management and impact. Subsequently, the estimation of LD is carried out according to the evolution of impact matrices toward higher impacts, where the Index of Agreement (IOA) and the Index of Symmetry (IS) should show that LDs have IOA close to one with respect to the reference LD, and where the IS with a high positive value indicate long tail distributions. Conceptually, the IOA stands on the work of Park and Seok [50]. According to these authors, the validation of an adaptation model applies the following seven statistical metrics that allow to unfold the IOA: Fractional Bias (FB), Normalized Mean Square Error (NMSE), Geometric Bias Mean (GBM), Geometric Bias Variance (GBV), Within a Factor of Two (FAC2), Unpaired Accuracy of Peak (UAPC) and Mean Relative Error (MRE).

\section{Experimental results}

\subsection{Learning process stage (on-line)}

Table 4 shows the flexibility of the FIAFIm with respect to the estimation of the LD_MC in the learning phase. In general, the model reached an average IOA (index of agreement) of 0.99689 for its three different forms (IUAFIm, IANUFIm, IASFIm) described in Section 3.4.1, with similar values for median, standard deviation, positive asymmetry, coverage indices $\left(C E L=E L / O p V a r_{99.9}\right.$, $C U L=U L / O p$ Varr $_{99.9}$ ) and number of tail data, preserving the structure (Generalized Pareto) and shape (long tail - positive asymmetry) of the estimated LDs, which provided evidence to support the invariance of the model with regard to the estimation of LD_MC.

Table 4: IC-Fingerprint for each form of the FIAFIm model estimating LD_MC in the learning phase

\begin{tabular}{lcccc}
\hline & LD_MC & LD_IUAFm & LD_IANUFm & LD_IASFm \\
\hline Distribution & G.Pareto & G.Pareto & G.Pareto & G.Pareto \\
\hline AIC & -3593.92 & -4234.75 & -2458.4 & -4176.4 \\
\hline shape $(k)$ & 0.176 & 0.188 & 0.187 & 0.186 \\
\hline scale $(\sigma)$ & 7.108 & 13.18 & 13.521 & 12.468 \\
\hline threshold $(\theta)$ & $-2.22 \mathrm{E}-15$ & $-2.22 \mathrm{E}-15$ & $-2.22 \mathrm{E}-15$ & $-2.22 \mathrm{E}-15$ \\
\hline kurtosis & 20.311 & 19.467 & 16.398 & 17.016 \\
\hline assymetry & 3.608 & 3.577 & 3.336 & 3.373 \\
\hline OpV $a R_{99.9 \%}$ & 105.16 & 179.416 & 179.418 & 160.993 \\
\hline EL & 8.636 & 16.254 & 16.635 & 15.33 \\
\hline UL & 96.524 & 163.162 & 162.783 & 145.663 \\
\hline CEL & 0.082 & 0.091 & 0.093 & 0.095 \\
\hline CUL & 0.918 & 0.909 & 0.907 & 0.905 \\
\hline TD & 321 & 317 & 317 & 316 \\
\hline EG & 0.308 & 0.303 & 0.302 & 0.301 \\
\hline IV & 3.237 & 5.43 & 5.424 & 4.845 \\
\hline IOA & 1 & 0.996 & 0.997 & 0.998 \\
\hline
\end{tabular}

Figure 10 shows the shape and distribution of fuzzy sets that make up the random linguistic variables of frequency and severity after the learning phase was carried out by the IANUFIm with respect to the estimation of LD_MC. In line with Definition 4, these fuzzy sets show that they are distributions with long tails, according to the slopes of the lines that establish the correlation between the quantiles $\left(q_{j}\right)$ and the normalized fuzzy sets centroids $\left(X C_{n, j, i}\right)$. In the case of the frequency, the value of the slope was $m_{f}=1.074$, while the value for severity was $m_{s}=1.8535$, which is in line with the general structure of the LD estimated by the model (long tail). 

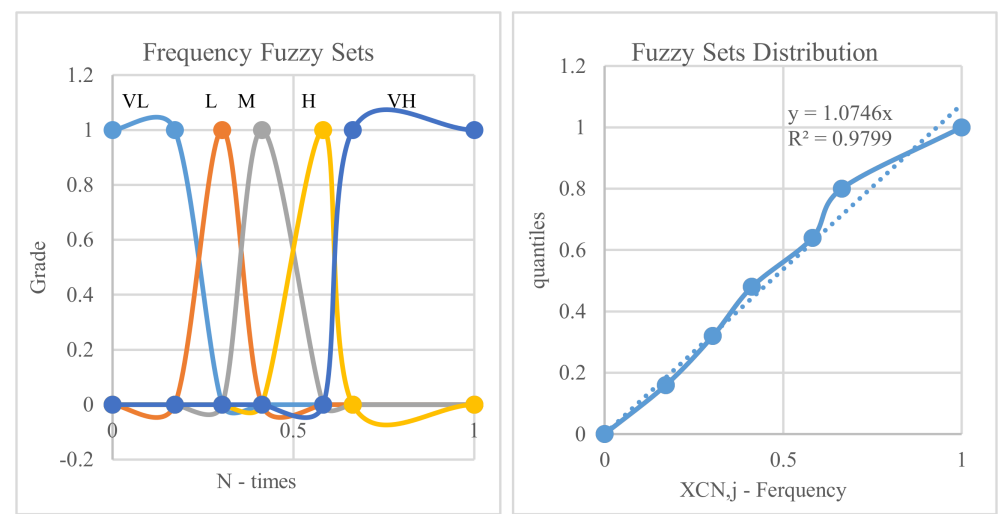

(a)

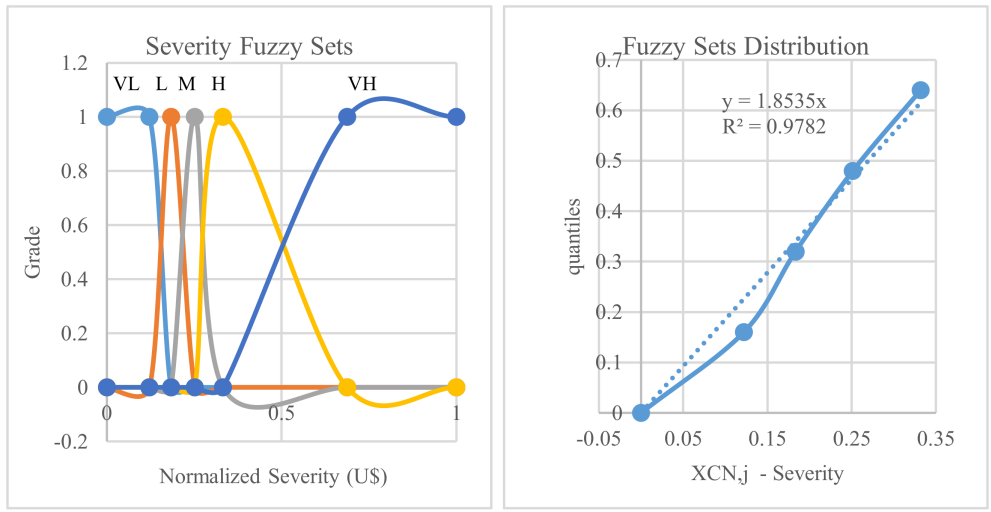

(b)

Figure 10: Fuzzy sets obtained as a result of the estimation process for LD_MC in the learning phase using the IANUFm: (a) Fuzzy sets of frequency (b) Fuzzy sets of severity

Figures 10 and 11 show that the fuzzy sets obtained by the IANUFIm and IASFIm, with regard to the estimation of LD_MC in the learning phase, had similar structures, with an IOA of 0.9915 and 0.9346 , between the normalized location of fuzzy sets that conform the random linguistic input variables for frequency and severity, respectively. These results again provide evidence of the invariance of the structure of the FIAFIm in estimating LD_MC through the application of two different behaviors.

Figure 12 shows the location of fuzzy sets, that define the main diagonal of the $T L M^{l}$, for each model that constitutes the general structure of FIAFIm. This fuzzy approach had similar structures and shapes as a result of the estimation of the LD_MC, evidenced by the slope values of $m_{I A U F m}=$ $0.8127, m_{I A N U F m}=0.4595$ and $m_{I A U S F m}=0.8904$, respectively. These slopes show that the fuzzy sets tended to be located to the right side of the horizontal axis, indicating higher values of risk has to be associated with higher values of management.

\subsection{Evolution process}

Table 5 shows the behavior of the IASFIm with respect to the estimation of the LDs for the sequence of risk after the learning process. Analyzing the contents of this table it can be observed that the indices $O p V a R_{99.9 \%}$, Scale $(\sigma)$, EL, UL and Insurance were decreasing along with the sequence of risk profiles: E1-E2-E3. The result is that the LDs will approach the LD_MC for the latter risk profile, which clearly shows the evolution of the LD towards lower values of risk due to the effect of a better risk management.

Figure 13 shows that despite of the evolution of the LD towards lower values, according to the aforementioned sequence of risk, the structure (Generalized Pareto) and shape (long tail - $\xi$ ) of the LD distributions remained. This fact makes the model ideal for assessing different risk management profiles in an organization in real time by applying its risk profile. This way, a delay in assessing the impact of risk management over time, which can be observed when applying traditional (non-fuzzy) models, is avoided. 

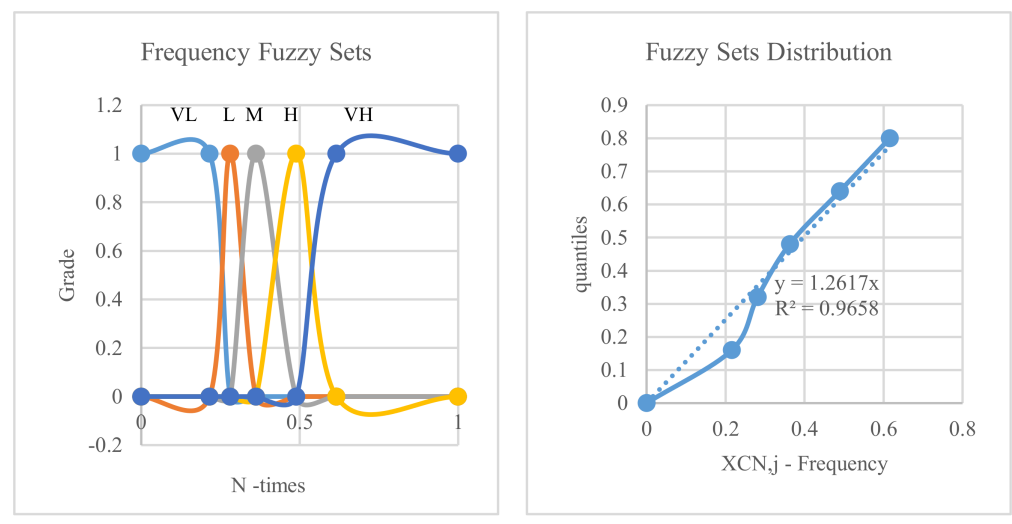

(a)

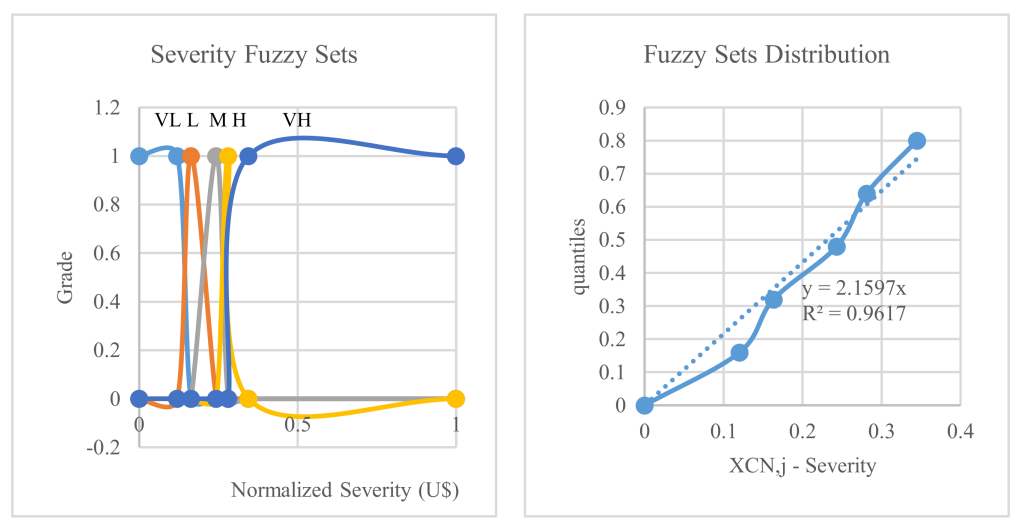

(b)

Figure 11: Fuzzy sets modelled for IASFm: (a) Fuzzy sets of frequency (b) Fuzzy sets of severity

Table 5: Evolution of the LD, estimated through the IASFm for the defined sequence of risk

\begin{tabular}{lccccc}
\hline & LDA_MC & LD_IAUSFm & LD_E3 & LD_E2 & LD_E1 \\
\hline Distribution & G.Pareto & Logistic & Logistic & Logistic & Logistic \\
\hline AIC & -3708.41 & -3554.12 & -4290.88 & -5342.16 & -5961.9 \\
\hline shape $(k)$ & 0.176 & 0.175 & 0.186 & 0.186 & 0.186 \\
\hline scale $(\sigma)$ & 7.108 & 6.838 & 13.981 & 39.828 & 74.427 \\
\hline threshold $(\theta)$ & $-2.22 \mathrm{E}-15$ & $-2.22 \mathrm{E}-15$ & $-2.22 \mathrm{E}-15$ & $-2.22 \mathrm{E}-15$ & $-2.22 \mathrm{E}-15$ \\
\hline kurtosis & 20.311 & 15.563 & 17.016 & 17.031 & 16.671 \\
\hline assymetry & 3.608 & 3.237 & 3.373 & 3.375 & 3.342 \\
\hline OpVaR $R_{99.9 \%}$ & 117.915 & 81.925 & 180.52 & 511.295 & 940.126 \\
\hline EL & 9.683 & 8.29 & 17.189 & 49.101 & 91.427 \\
\hline UL & 108.232 & 73.635 & 163.331 & 462.195 & 848.699 \\
\hline CEL & 0.082 & 0.101 & 0.095 & 0.096 & 0.097 \\
\hline CUL & 0.918 & 0.899 & 0.905 & 0.904 & 0.903 \\
\hline TD & 321 & 320 & 316 & 316 & 316 \\
\hline EG & 0.308 & 0.304 & 0.301 & 0.301 & 0.301 \\
\hline IV & 3.63 & 2.489 & 5.433 & 15.381 & 28.263 \\
\hline IOA & 1 & 0.997 & 0.998 & 0.998 & 0.998 \\
\hline
\end{tabular}



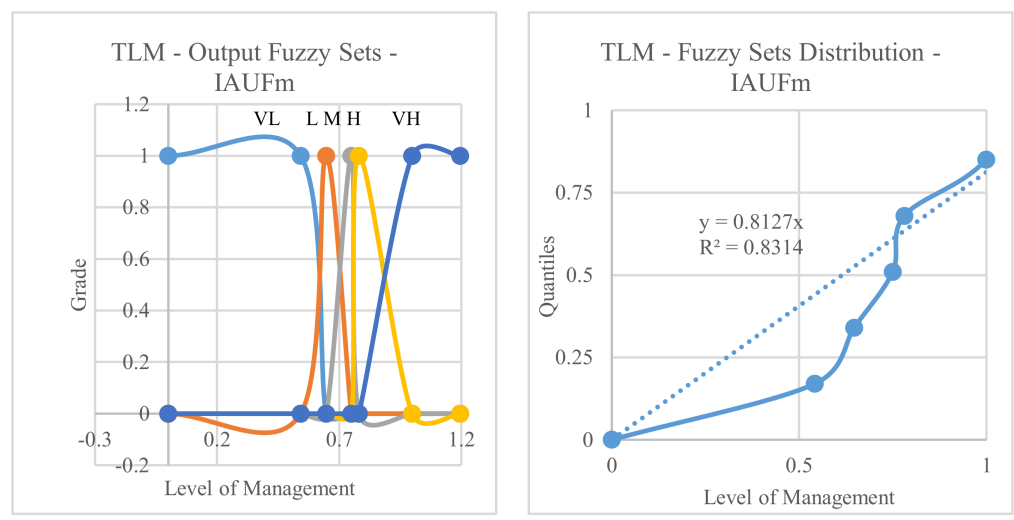

(a)
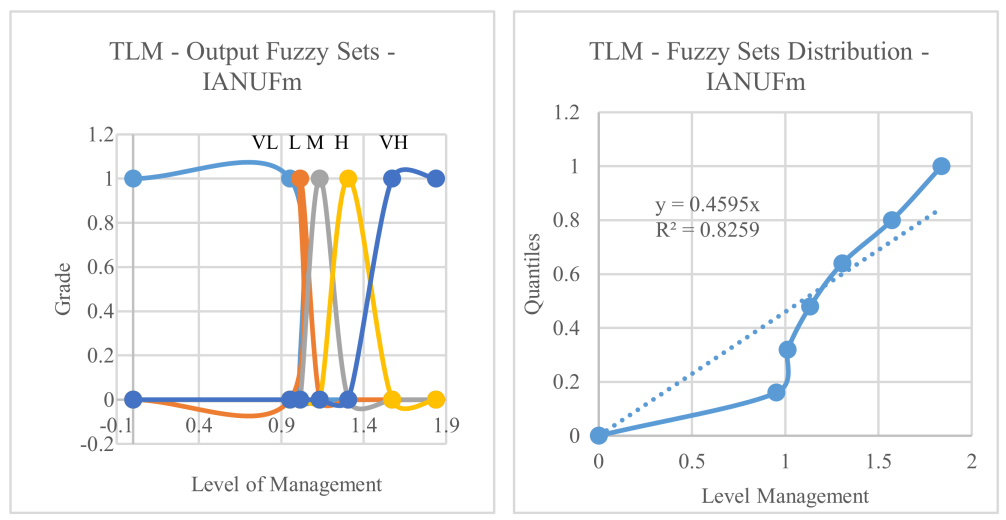

(b)

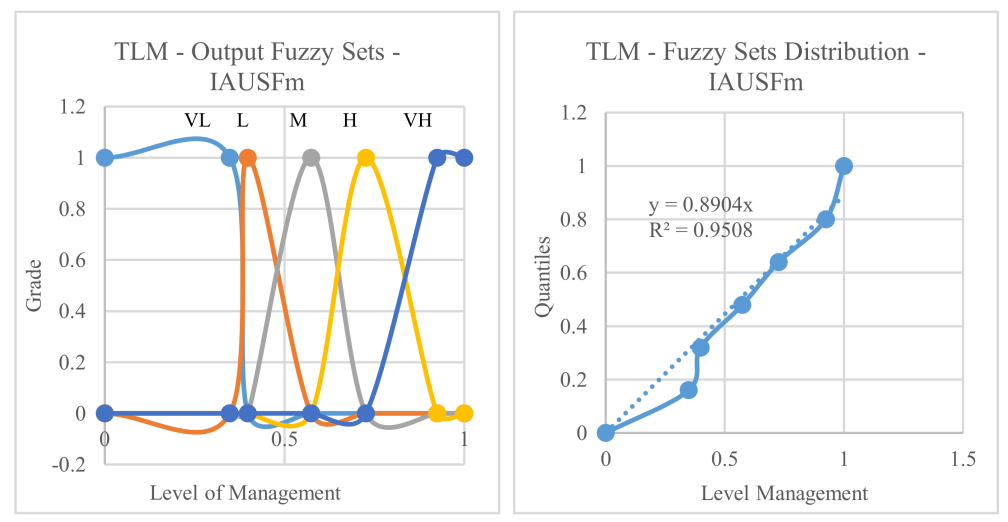

(c)

Figure 12: Output - fuzzy sets obtained through the $T L M^{l}$ as a result of the estimation of LD reference: (a) IAUFm (b) IANUFm (c) IASFm 


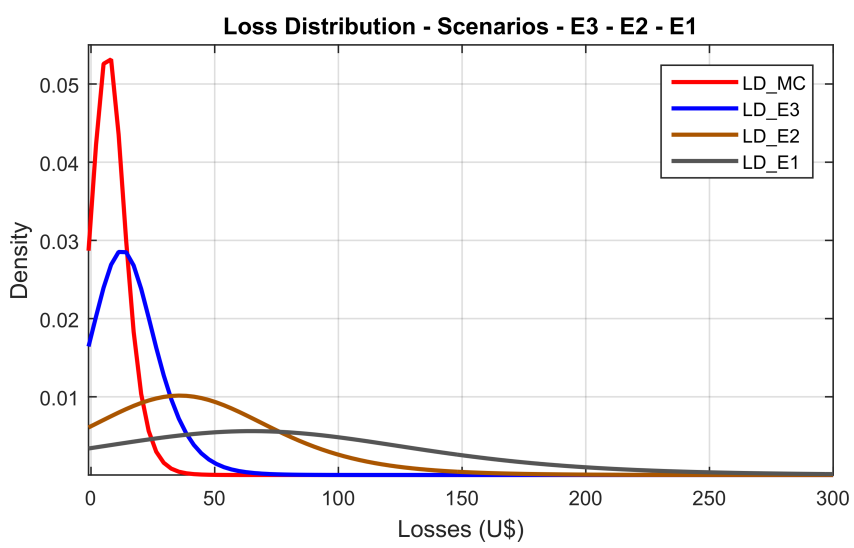

(a)

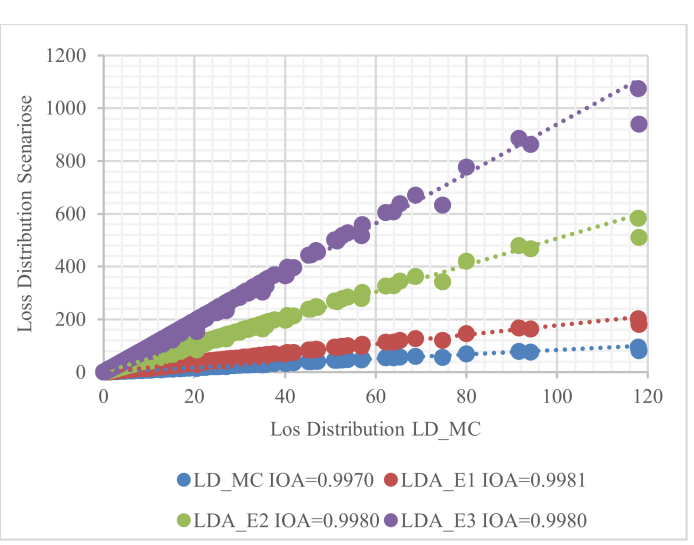

(b)

Figure 13: LDA distributions obtained by the model after the process of estimating of a reference LDA for the sequence E3-E2-E1 (a) LDs; (b) IOAs

Table 6: Validation metrics and obtained values for the experimental results

\begin{tabular}{l|c|c|c|c|c|c|c|c}
\hline & LD_IAUSFm & \multicolumn{2}{|c|}{ LD_E3 } & \multicolumn{2}{c|}{ LD_E2 } & \multicolumn{2}{c}{ LD_E1 } \\
\hline FB & 0.139534 & G & -0.519910 & G & -1.296955 & P & -1.585551 & P \\
\hline NMSE & 0.028883 & G & 0.319436 & G & 3.123934 & G & 7.215702 & F \\
\hline GBM & 1.129573 & G & 0.628853 & G & 0.246637 & G & 0.137126 & P \\
\hline GBV & 1.016517 & G & 1.256042 & G & 7.608656 & F & 57.913736 & P \\
\hline FAC2 & 1.000000 & G & 1.000000 & G & 0.029000 & P & 0.014000 & P \\
\hline UAPC & 0.200349 & G & -0.713737 & G & -3.906609 & G & -8.036214 & G \\
\hline MRE & 0.113156 & G & -0.598225 & G & -3.159291 & P & -6.572357 & P \\
\hline IOA & 0.997 & G & 0.998 & G & 0.998 & OF & 0.998 & P \\
\hline Score & 68.0 & & 68.0 & & 45.0 & & 29.5 & \\
\hline
\end{tabular}

For the analysis and validation of the proposed model with regard to the estimation of the loss distribution according to the evolution of the impact matrices, it can be observed that the IOA and the IS show that the distributions of reference losses maintain their structure and shape in terms of the LDs obtained for each impact matrix. According to Park and Seok [50], the validation of an adaptation model applies the seven statistical metrics mentioned in the methodology section to unfold the IOA. To obtain the performance (score) value of a model, Park and Seok [50] propose a series of values that are additive according to the qualities taken by each metric as follows: Good 7-10 (average 8.5), Fair 4-7 (average 5.5), OverFair (average 6), UnderFair (average 5) and Poor 1-4 (average 2.5). The score of a model is computed as follows:

$$
\text { Score }=\sum_{i=1}^{8} q\left(\text { Scale }_{i}\right)
$$

where $q\left(\right.$ Scale $\left._{i}\right)$ is the corresponding average value in the set $\{8.5,5.5,6,5,2.5\}$ associated to each one of the classes \{Good, Fair, OverFair, UnderFair, Poor\}, respectively, that the metric $i \in\{1, \ldots, 8\}$, which refers to the 8 metrics $\{\mathrm{FB}$, NMSE, GBM, GBV, FAC2, UAPC, MRE, IOA $\}$, takes. The maximum score value that a model can achieve is 68 points.

According to Table 6, the stability of the model for the estimation of the LD can be observed for different management scenarios. This stability is a consequence of only small variations experienced by the IOA and IS, which guarantees long tail distributions of the LDs representing each impact matrix. According to the IOA, the proposed model achieved an underestimation of the data close to $14 \%$ in the adaptation and learning stage, as shown by the FB index. In this first stage the management and impact matrices are similar in magnitude as can be seen in Figure 13. It is important to mention that 
Table 7: Evolution of tail data in the LD as a result of a better risk management

\begin{tabular}{lccccc}
\hline & LD_MC & LD_IAUSFm & LD_E3 & LD_E2 & LD_E1 \\
\hline Distribution & G.Pareto & G.Pareto & G.Pareto & G.Pareto & G.Pareto \\
\hline AIC & -3708.410 & -3554.120 & -4290.880 & -5342.160 & -5961.900 \\
\hline shape $(k)$ & 0.176 & 0.175 & 0.186 & 0.186 & 0.186 \\
\hline scale $(\sigma)$ & 7.108 & 6.838 & 13.981 & 39.828 & 74.427 \\
\hline threshold $(\theta)$ & $-2.2204 \mathrm{E}-15$ & $-2.22 \mathrm{E}-15$ & $-2.22 \mathrm{E}-15$ & $-2.22 \mathrm{E}-15$ & $-2.22 \mathrm{E}-15$ \\
\hline OpVaR $R_{99.9 \%}$ & 117.915 & 81.925 & 180.520 & 511.295 & 940.126 \\
\hline Tail data (un) & 1.000 & 1.000 & 8.000 & 100.000 & 240.000 \\
\hline
\end{tabular}

this value became increasingly negative as the impact matrices took higher values, which evidences the importance of management and its impact. This behavior is also reflected in the behavior of the GBM index, which shows the difference between the mean of the reference distribution and the mean of the distributions obtained for each scenario.

With respect to extreme events, on one hand it can be said that the GBV increased as the impacts increase, which shows that a matrix with a greater magnitude of this index generates a heavier tail with a much higher OpVaR. A higher OpVaR is corroborated by an equally increasing NMSE index. On the other hand, the UAPC2 index shows the discrepancy between the OpVaR values at 99.9\%, associated to each LD obtained, delivering decreasing values that are increasingly negative. This means an increase of the OpVaR for higher impacts. The FAC2 index shows that most of the data for the highest impact matrices, which were $97.1 \%$ and $98.6 \%$ for LD_E2 and LD_E3, respectively, are mostly above $80 \%$ and $120 \%$ of the magnitude of the losses of the reference LD. In this way, the IOA and IS confirm research carried by Gnedenko et al. [48], which indicates that probability distributions with particular high IS and GBV values tend to be Pareto generalized with heavier tails. With respect to the score that defines the fuzzy model, as proposed by Park and Seok [50] to evaluate the performance of a model, an increase of the Score representing the evolution of the OpVaR in an organizations due to better risk management. That is, better risk management leads to impact matrices of much smaller magnitude that are close to the reference management matrices, which have been suggested by the Basel II regulations for this type of risk.

Figure 14 and Table 7 show the behavior experienced by the tail of the LD estimated by the IASFm, which is in agreement with the sequence established by the evolution of risk management. With regard to the $O p V_{a r} r_{99.9 \%}$ estimated from LD_MC, the tail of the distribution evolve towards longer and heavier tails with lower quantity of data, which can be explained to the pressure generated by a better risk management. This is consistent with the EVT (extreme value theory) that defines the estimation of Fuzzy OpVar.

This evolution process makes the model ideal for assessing a priori the evolution of operational risk in a financial organization, using different risk profiles. This helps to overcome both the restrictions imposed by the sampling of frequency and severity linguistic variables using the Montecarlo method and the restrictions imposed by the time that is required when using the traditional AMA models to assess the impact of a risk profile RMM on the LD that represents a risk event in a business line in a financial organization.

Table 8 shows the behavior of the model with different structures (IAUFm, IANUFm, IASFm) with regard to the estimation of the LD for risk profile E3. The statistical indices clearly show that the three structures of the FIAFIm model reached an IOA close to its maximum value of 1, preserving the structure (Generalized Pareto distribution) and shape (long tail), as well as the main statistical characteristics of the LD_MC. Figure 15 shows that the model can achieve similar behavior as the LD_MC due to the effect of a better risk management. This means that international standards as defined by the BCBS_Basel II can be achieved. 


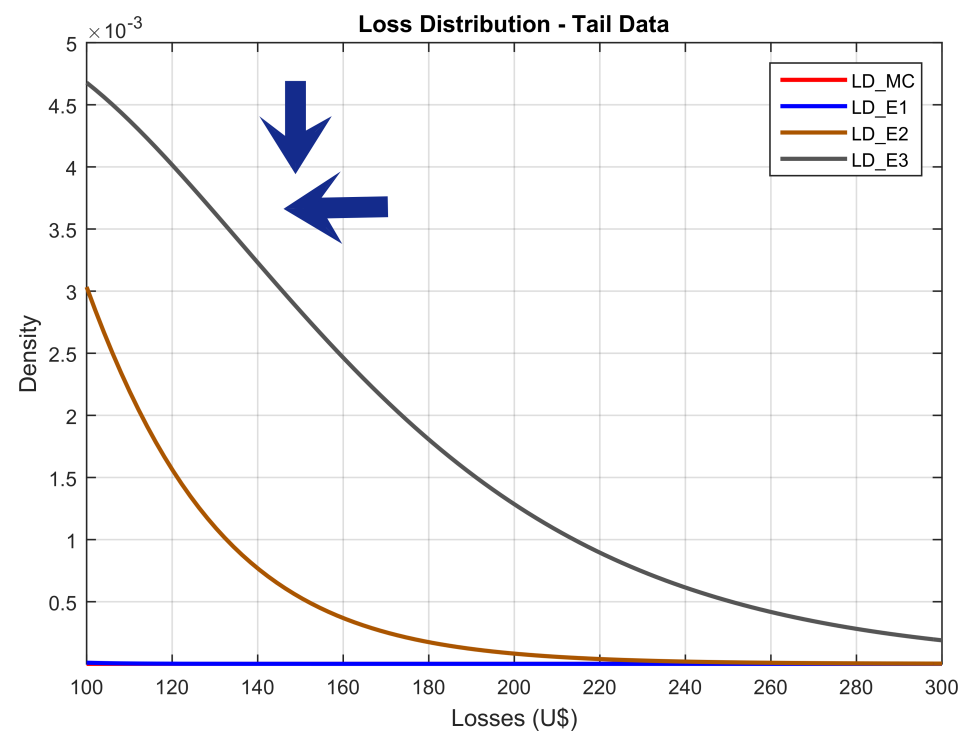

Figure 14: Behavior of the tail data for each scenario of risk with reference to LD_MC

Table 8: Behavior of the IAUFm, IANUFm and IASFm with respect to estimating the LD for risk profile E3

\begin{tabular}{lcccc}
\hline & LD_MC & LD_IAUFm & LD_IANUFm & LD_IASFm \\
\hline Distribution & G.Pareto & G.Pareto & G.Pareto & G.Pareto \\
\hline AIC & -3593.92 & -5273.98 & -5323.17 & -5227.67 \\
\hline shape $(k)$ & 0.176 & 0.185 & 0.186 & 0.189 \\
\hline scale $(\sigma)$ & 7.108 & 37.488 & 39.284 & 35.519 \\
\hline threshold $(\theta)$ & $-2.22 \mathrm{E}-15$ & $-2.22 \mathrm{E}-15$ & $-2.22 \mathrm{E}-15$ & $-2.22 \mathrm{E}-15$ \\
\hline kurtosis & 20.311 & 19.016 & 16.075 & 17.031 \\
\hline asymmetry & 3.608 & 3.538 & 3.309 & 3.375 \\
\hline OpV $a R_{99.9 \%}$ & 105.16 & 503.3 & 513.754 & 455.986 \\
\hline EL & 8.636 & 46.052 & 48.274 & 43.789 \\
\hline UL & 96.524 & 457.248 & 465.48 & 412.197 \\
\hline CEL & 0.082 & 0.092 & 0.094 & 0.096 \\
\hline CUL & 0.918 & 0.908 & 0.906 & 0.904 \\
\hline TD & 321 & 317 & 318 & 316 \\
\hline EG & 0.308 & 0.302 & 0.303 & 0.301 \\
\hline IV & 3.237 & 15.225 & 15.57 & 13.717 \\
\hline IOA & 1.000 & 0.996 & 0.997 & 0.998 \\
\hline & & & &
\end{tabular}




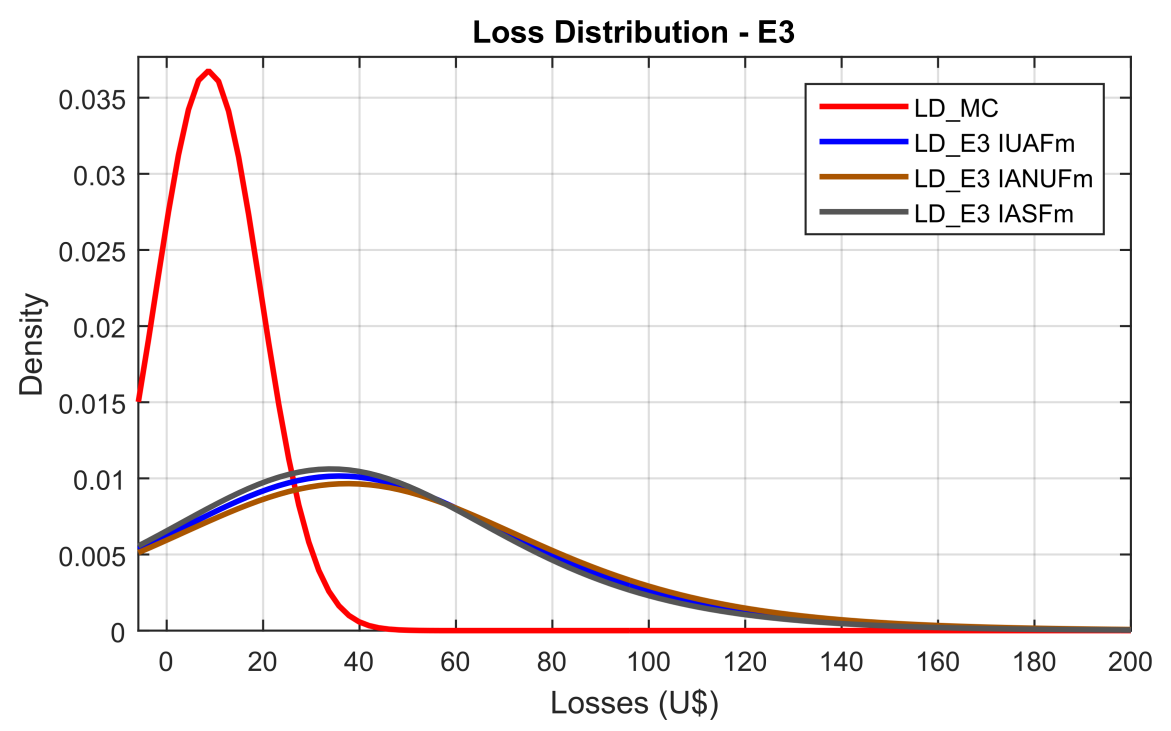

Figure 15: LD distributions obtained via IAUFm, IANUFm, IASFm using the RMM for risk profile E3-Soft Impact

\section{Conclusions and future work}

This paper presents a Flexible Inverse Adaptive Fuzzy Inference model, which integrates in a single model both a novel Montecarlo sampling method for linguistic variables that define the frequency and severity as input variables and different RMMs that show a priori the evolution of risk management in financial organizations and its impact on the OpVaR. This model overcomes the limitations imposed by traditional AMA models that require a period of time to evaluate this impact.

Due to the flexible inverse adaptive fuzzy inference model, the proposed FIAFIm model in its different forms (IAUFIm-Inverse Adaptive Uniform Fuzzy Inference Model; IANUFIm-Inverse Adaptive Non Uniform Fuzzy Inference Model; IASFIm-Inverse Adaptive Sampling Fuzzy Inference Model) allows, in a learning phase, to identify both the random input variables for frequency and severity by using the structure and shape of fuzzy sets, and the $T L M^{l}$, which shows the true levels of risk management in an organization.

We used the outcome of the learning phase to analyze these models in terms of the impact that different risk profiles generate on the LD. The unfolding of the IOA based on the seven fuzzy statistical metrics shows the reduction of OpVaR values. Due to the shown stability of the model based on the IOA values, the loss distributions adjust to Generalized Pareto. The model achieves this stability and the autonomy in the estimation of the OpVaR without using adaptation and learning mechanisms. The decreasing values for the GBM and GBV lead to long tail distributions, which is typical for operational risk. With regard to the risk management, we conclude that it is inversely proportional to the OpVaR, which means that the RMMs with a higher level of risk management lead to lower OpVaR values.

The experimental results showed that the models IANUFIm and IASFIm achieved similar results with regard to the identification of the current risk management in an organization as well as with respect to the structure and shape of the input and output of fuzzy sets in different profiles of risk. This means that the model showed an effective Montecarlo behavior in producing a new inverse fuzzy model with statistical behavior. This reinforces the validity of the fuzzy approach that has been put forward in this paper.

With respect to future work, the authors consider interesting and promising to research in more detail about identifying inherited risk matrices that show the profile of an organization with regard to their risk management. A solution might be achieved by integrating the behavior of the Montecarlo sampling method with an ANFIS model.

\section{Acknowledgements}

The authors would like to thanks the anonymous referees for providing constructive comments and suggestions. 


\section{References}

[1] Bank for International Settlements, Recongising the risk mitigating impact of insurance in operational risk modelling, http://www.bis.org/publ/bcbs181.htm (October 2010).

[2] A. Mora Valencia, Cuantificación del riesgo operativo en entidades financeiras en colombia, Cuadernos de Administración 25 (41) (2010) 185-211.

[3] F. Z. El Arif, S. Hinti, Mehtods of quantifying operational risk in banks: Theoretical approaches, American Jounral of Engineering Research 03 (03) (2014) 238-244.

[4] A. Reveiz, C. León, Operational risk management using a fuzzy logic inference system, Borradores de Economía 574, Banco de la República Colombia (2009).

[5] P. X. Girling, Operational risk management: a complete guide to a successful operational risk framework, John Wiley \& Sons, 2013.

[6] E. Lee, Y. Park, J. G. Shin, Large engineering project risk management using a bayesian belief network, Expert Systems with Applications 36 (2009) 5880-5887.

[7] L. B. Andersen, D. Hager, S. Maberg, M. B. Naess, M. Tungland, The financial crisis in an operational risk management context - a review of causes and influencing factors, Reliability Engineering \& System Safety 105 (2012) 3-12.

[8] S. Figini, L. Gao, P. Gindici, Bayesian operational risk models, DEM Working papers series 10 (1) (2015) 7-13.

[9] S. Barua, X. Gao, H. Pasman, S. Mannan, Bayesian network based dynamic operational risk assessment, Journal of Loss Prevention in the Process Industries 41 (2016) 399-410.

[10] B. Twala, Multiple classifier application to credit risk assessment, Expert Systems with Applications 37 (2010) 3326-3336.

[11] L. Yu, W. Yue, S. Wang, K. K. Lai, Suport vector machine based multiagent ensemble learning for credit risk evaluation, Expert Systems and Applications 37 (2010) 1351-1360.

[12] L. Yu, X. Yao, S. Wang, K. K. Lai, Credit risk evaluation using a weighted least squares svm classifier with design experim for parameter selection, Expert Systems Applications 38 (2011) $15392-15390$.

[13] A. Feki, A. Ben Ishak, S. Feki, Feature selection using bayesian and multiclass support vector machines approaches: Application to bank risk prediction, Expert Systems with Applications 39 (2012) 3087-3099.

[14] L. Yu, Credit risk evaluation with least squares fuzzy support vector machines classifier, Discrete Dynamics in Nature and Society 2014, Article ID 564213 (2014) 9 pages.

[15] K. Ye, J. Yan, S. Wang, H. Wang, B. Miao, Knowledge level modeling for systematic risk management infinancial institutions, Expert Systems with Applications 38 (2011) 3528-3538.

[16] A. S. Koyuncugil, N. Ozgulbas, Financial early warning system model and data mining application for risk detection, Expert Systems with Applications 39 (2012) 6238-6253.

[17] J. A. Hernández, J. D. Opsina, A multi dynamics algorithm for global optimization, Mathematical and Computer Modelling 5 (7-8) (2010) 1271-1278.

[18] P.-C. Lin, P.-C. Ko, Portfolio value at risk forecasting with ga-based extreme value theory, Expert Systems with Applications 36 (2009) 2503-2512.

[19] D. D. Pinto, J. G. M. S. Monteiro, E. H. Nakao, An approach to portfolio selection using arx predictor for securities' risk and return, Expert Systems with Applications 38 (2011) 15009-15013. 
[20] C. M. Lopera, M. C. Jaramillo, L. D. Arcila, Selection of a copula model to fit bivariante depend data, Dyna 76 (158) (2009) 253-263.

[21] P. Dorogovs, I. Solovjova, A. Romanovs, New tendencies of management and control of operational risk in financial institutions, Procedia - Social and Behavioral Sciences 99 (6) (2013) 911-918.

[22] L. Koliali, Extreme risk modeling: An evt-pair copulas approach for financial stress test, Journal of Banking \& Finance 70 (2016) 1-22.

[23] M. Hadjimichael, A fuzzy expert system for aviation risk assessment, Expert Systems with Applications 36 (2009) 6512-6519.

[24] A. Khashman, Neural networks for credit risk evaluation: Investigation of different neural models and learning schemes, Expert Systems with Applications 37 (2010) 6233-6239.

[25] A. Golmohammadi, M. Pajoutan, Metaheuristics for dependent portfolio selection problem considering risk, Expert Systems with Applications 38 (2011) 5642-5649.

[26] Y. Deng, R. Sadiq, W. Jiang, S. Tesfamariam, Risk analysis in a linguistic environment. a fuzzy evidential reasoning-based approach, Expert Systems with Applications 38 (2011) 15438-15446.

[27] K. Mokhtari, J. Ren, C. Roberts, J. Wang, Decision support framework for risk management on sea ports and terminals using fuzzy set theory and evidential reasoning approach, Expert Systems with Applications 39 (2012) 5087-5103.

[28] B. Cooper, B. Piwcewicz, N. Warren, Operational risk modelling: how fare we progressed, in: Financial Services Forum, Actuaries Institute, 2014, pp. 2-27.

[29] S. Mitra, A. Karathanasopoulos, G. Sempinis, C. Dunis, Operational risk: emerging markets, sectors and measurement, European Jounral of Operational Research 24 (1) (2016) 122-132.

[30] F. Chiclana, F. Mata, L. G. Pérez, E. Herrera-Viedma, Type-1 OWA unbalanced fuzzy linguistic aggregation methodology: Application to eurobonds credit risk evaluation, International Journal of Intelligent Systems doi:10.1002/int.21912.

[31] S. Shah, Measuring and managing operational risks (2002). URL http://tinyurl.com/shah02measuring

[32] S. Shah, Measuring operational risks using fuzzy logic modeling (2003).

URL http://tinyurl.com/shah03measuring

[33] M. G. Cruz, G. W. Peters, P. V. Shevchenko, Fundamental aspects of operational risk and insurance analytics: A handbook of operational risk, John Wiley \& Sons, 2015.

[34] M. Takács, Soft Computing-Based Risk Management-Fuzzy, Hierarchical Structured DecisionMaking System, InTech, 2011, Ch. 2, pp. 27-46.

[35] K. Shang, Z. Hossen, Applying fuzzy logic to risk assessment and decision-making, Tech. rep., Casualty Actuarial Society, Canadian Institute of Actuaries, Society of Actuaries (2013).

[36] M. Mainelli, System Dynamics Und Key Risk Indicators - PKRI-LI Im Wholesale Banking, Frankfurt School Verlag, 2008, Ch. 9, pp. 439-456.

[37] A. D. Sanford, I. A. Moosa, A bayesian network structure for operational risk modelling in structured finance operations, Journal of the Operational Research Society 63 (4) (2012) 431444.

[38] Austrian OeNB and FMA, Guidelines on operational risk management, http://tinyurl.com/ ljnxa5h (2006). 
[39] P. Otero, O. Veneiro, Determinación del requerimiento de capital por riesgo operacional Metodología "value at risk", Quantum 4 (1) (2009) 58-79.

[40] Bank for International Settlements, Principles for the sound management of operational risk, http://www.bis.org/publ/bcbs195.htm (June 2011).

[41] HSBC, Riesgo operacional, Tech. rep., HSBC México (HBMX) (2007).

[42] A. Jobst, The treatment of operational risk under the new based framework: Critical issues, Journal of Banking 8 (4) (2007) 316-352.

[43] Goverment of Canada, Guide to Corporate Risk Profiles (Basel II), https://www.tbs-sct.gc . ca/hgw-cgf/pol/rm-gr/gcrp-gepro/gcrp-gepropr-eng. asp (2011).

[44] ISO, ISO 31000 - Risk Management, ISO-ITC-UNIDO, Geneva Switzerland, 2015.

[45] P. Carbone, J. Schoukens, I. Kollar, A. Moschitta, Measuring the noise cumulative distribution function using quantized data, IEEE Transactions on Instrumentation and Measurement 65 (2016) $1540-1546$

[46] A. Peña P, J. Hernández R, Construction of PMx concentration surfaces using neural evolutionary fuzzy models of type S, Studies in Computational Intelligence 628 (2016) 341-369.

[47] D. E. Rumelhart, R. J. Hinton, Geoffrey E.and Williams, Learning representations by backpropagating errors, Nature 323 (1986) 533-536.

[48] B. V. Gnedenko, K. Yu, A. Belyayev, D. Solovyev, Z. W. Bimbaum, E. Luckacs, Mathematical Methods of Realibiality Theory, Academic Press, 1969.

[49] F. De Martino, F. Gentile, F. Esposito, M. Balsi, F. Di Salle, R. Goebel, E. Formisano, Classification of fmri independent components using ic-fingerprints and support vector machine classifiers, NeuroImage 34 (1) (2007) 177-194.

[50] O.-H. Park, M.-G. Seok, Selection of an appropriate model to predict plume dispersion in coastal areas, Atmospheric Environment 41 (29) (2007) 6095-6101. 